\title{
Tetrahedral-Mesh Simulation of Turbulent Flows with the Space-Time Conservative Schemes
}

\author{
Chau-Lyan Chang, \\ NASA Langley Research Center, Hampton, VA 23681 \\ Balaji Venkatachari ${ }^{\dagger}$ \\ National Institute of Aerospace, Hampton, VA 23666 \\ and \\ Gary C. Cheng \\ University of Alabama, Tuscaloosa, AL 35401
}

Direct numerical simulations of turbulent flows are predominantly carried out using structured, hexahedral meshes despite decades of development in unstructured mesh methods. Tetrahedral meshes offer ease of mesh generation around complex geometries and the potential of an orientationfree grid that would provide un-biased small-scale dissipation and more accurate intermediate scale solutions. However, due to the lack of consistent multi-dimensional numerical formulations in conventional schemes for triangular and tetrahedral meshes at the cell interfaces, numerical issues exist when flow discontinuities or stagnation regions are present. The space-time conservative conservation element solution element (CESE) method - due to its Riemann-solver-free shock capturing capabilities, non-dissipative baseline schemes, and flux conservation in time as well as space - has the potential to more accurately simulate turbulent flows using unstructured tetrahedral meshes. To pave the way towards accurate simulation of shock/turbulent boundary-layer interaction, a series of wave and shock interaction benchmark problems that increase in complexity, are computed in this paper with triangular/tetrahedral meshes. Preliminary computations for the normal shock/turbulence interactions are carried out with a relatively coarse mesh, by direct numerical simulations standards, in order to assess other effects such as boundary conditions and the necessity of a buffer domain. The results indicate that qualitative agreement with previous studies can be obtained for flows where, strong shocks co-exist along with unsteady waves that display a broad range of scales, with a relatively compact computational domain and less stringent requirements for grid clustering near the shock. With the space-time conservation properties, stable solutions without any spurious wave reflections can be obtained without a need for buffer domains near the outflow/farfield boundaries. Computational results for the isotropic turbulent flow decay, at a relatively high turbulent Mach number, show a nicely behaved spectral decay rate for medium to high wave numbers. The high-order CESE schemes offer very robust solutions even with the presence of strong shocks or widespread shocklets. The explicit formulation in conjunction with a close to unity theoretical upper Courant number bound has the potential to offer an efficient numerical framework for general compressible turbulent flow simulations with unstructured meshes.

\section{Nomenclature}

$\begin{array}{ll}A & =\text { area of the space-time element interface } \\ d p & =\text { normalized pressure ratio } \\ e & =\text { total energy, defined in the second section } \\ E & =\text { turbulent kinetic energy } \\ F & =\text { flux vectors for general conservation laws }\end{array}$

\footnotetext{
*Aerospace Technologist, Computational AeroSciences Branch, Email: Chau-Lyan.Chang@,nasa.gov, Associate Fellow, AIAA

${ }^{\dagger}$ Research Engineer, Email: balaji.s.venkatachari@nasa.gov, Senior member, AIAA

*Associate Professor, Department of Aerospace Engineering and Mechanics, Email: gary.cheng@eng.ua.edu, Associate Fellow, AIAA
} 


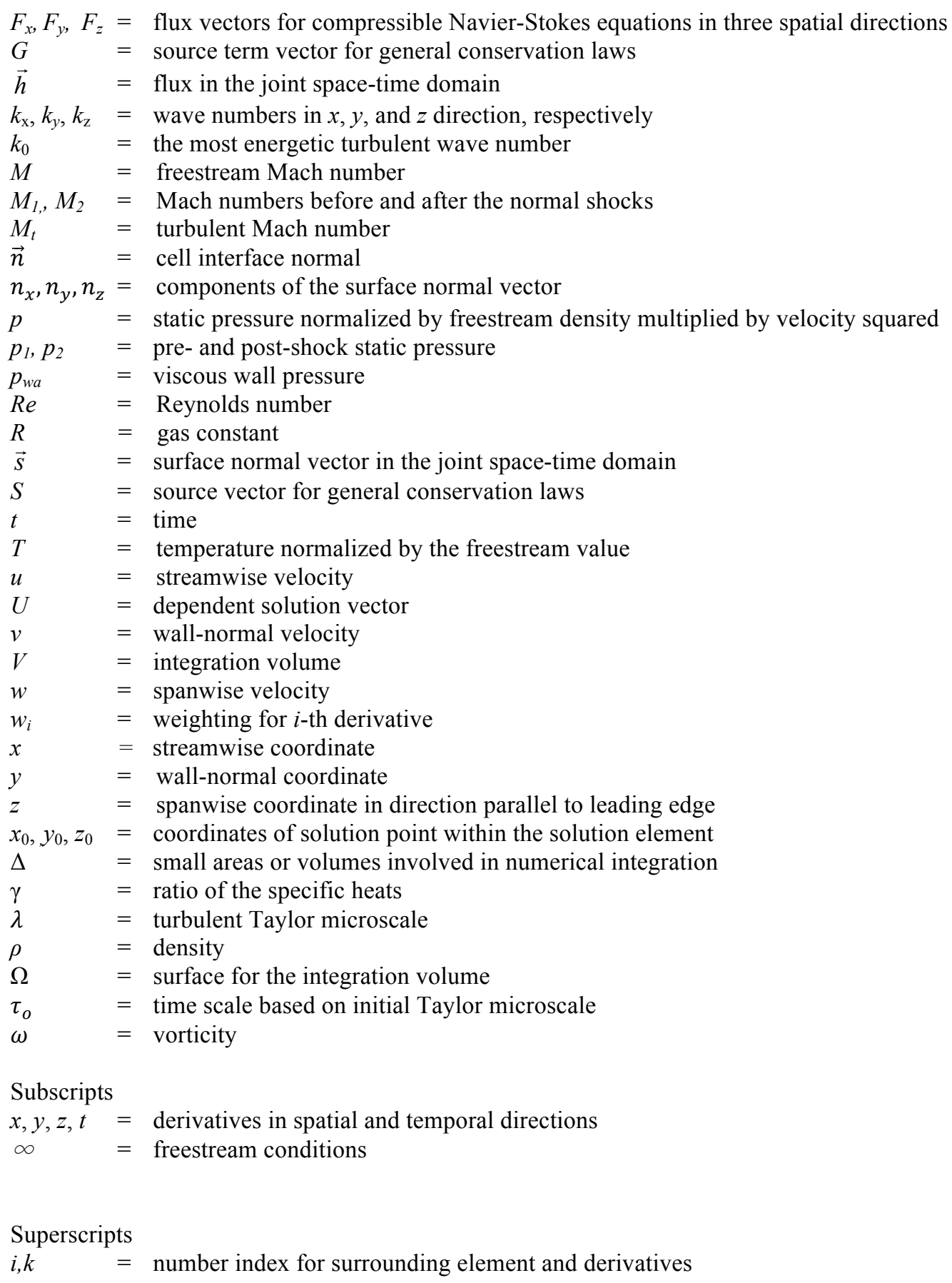

\section{Introduction}

Numerical simulations of turbulent flows have been actively pursued for several decades. Parallel to the advancement of CPU speed and large-scale parallel clusters, mesh size for turbulent flow simulations is approaching trillions of grid points. ${ }^{1}$ The large-scale parallel computations done in Ref. 1 simulated isotropic turbulent flows interacting with shocks by using about 2 million CPU cores. Excellent parallel scaling with low memory footprint for structured meshes was demonstrated using space-time explicit $6^{\text {th }}$ order central-difference or $5^{\text {th }}$-order weighted essentially non-oscillatory (WENO) schemes. According to the authors, the achievement of nearly linear scaling up to about 2 million processes is mainly attributed to 
the explicit time-marching in conjunction with non-blocking MPI communications. ${ }^{1}$ The low memory footprint for structured meshes avoids memory access across the different ports within the node, thereby allowing hyperthreading to speed up the computations without incurring memory overhead.

A lot can be learned from this one of a kind simulation. Implicit schemes have been preferred for many CFD computations due to their robustness to deal with stiffness (either from embedded poor-quality mesh elements or stiff source terms) and fast convergence to steady state solutions. For large-scale nonRANS (Reynolds Average Navier-Stokes) turbulent flow simulations (and transitional flow or acousticwave computations), however, implicit schemes lose their edge when the solution accuracy rather than the convergence rate to steady state dictates the time step. Two aspects make implicit schemes less competitive for such time-accurate computations. Firstly, to preserve time accuracy, sub-iterations have to be employed. Its accompanying cost cannot be compensated by using a larger time step, due to accuracy consideration. Secondly, as the mesh size increases to the order of billions or trillions, the solution time required to solve the accompanying matrices in implicit schemes also increases significantly. Preconditioning or other accelerating algorithms could be used to reduce the computational time. Nevertheless, the increased matrix size makes the memory usage suboptimal under the large-scale cluster environment. Parallel scaling for banded matrices has been poor for the current computer architecture. From a flow physics standpoint, the physical time scale in most unsteady, transitional, or turbulent flows is quite close to that of the acoustic wave. The vorticity or other viscous modes near the wall may have a substantially longer time scale than the corresponding acoustic waves. This implies that the time step determined from the CFL number of about 1 is very close to the physical time scale. For such problems, explicit schemes free of matrix solvers with high-order time accuracy become more efficient than implicit schemes with the same time step size.

The low memory footprint for the above-mentioned simulations is unique to structured meshes. When an unstructured mesh is used, additional memory is required for connectivity and geometry information. Accessing neighboring cells becomes much more memory and communication intensive operations due to less orderly data around neighbors. Moreover, the required communication maps among MPI processes grow substantially when the mesh size becomes larger, and the decomposed domains may be surrounded by many other neighboring domains. Despite these shortcomings, unstructured meshes are still preferred for complex geometries due to their fewer topological constraints and ease of generation around corners and gaps. For turbulent flow simulations, unstructured meshes may offer another advantage. Triangular and tetrahedral mesh elements that are isotropic are free of artificial orientations. Such isotropic meshes in conjunction with proper (non-excessive) numerical dissipation could improve solution accuracy of medium-to-small scales in direct numerical simulations (DNS) or subgrid-scale models in large eddy simulations (LES). From this perspective, the ideal mesh for turbulent flow simulations is likely to be a nearly-isotropic tetrahedral mesh with cells that vary in size.

In light of the discussion above, efficient simulations for turbulent flows hinge upon tailoring MPI codes with hardware architectures as well as accurate numerical algorithms. With the success of pushing the state-of-the-art for structured-mesh simulations, more work is needed to explore the use of unstructured meshes and the required numerical algorithms to improve the accuracy while maintaining the key features of efficient large-scale simulations under modern parallel architectures. Along this line, this paper investigates numerical simulations of turbulent flows using explicit, strongly conservation property preserving schemes with isotropic tetrahedral meshes. Similar research has been going on around the world. ${ }^{2,3}$ For example, Khalighi et al. ${ }^{2}$ investigated time-explicit, hybrid algorithms for unstructured meshes and validated their algorithms and codes for turbulent jets and the accompanying acoustic wave propagation. In the present research, the space-time conservation element, solution element (CESE) method $^{5-8}$ is used for turbulent flow simulations with tetrahedral meshes. Turbulent flow simulation is investigated from the perspective of four unique aspects. Firstly, the CESE method is formulated by enforcing the strong space-time unity integral form of the conservation laws. ${ }^{5,9-10}$ A high degree of conservation is in general a key aspect in preserving numerical accuracy for waves and turbulent flow simulations. The flux conservation in time introduced in the CESE method, in particular, could offer appreciable advantages in turbulent flow computations. Secondly, the time-accurate local time-stepping (TALTS) scheme $e^{7-8,11}$ formulated based on the CESE method is appealing for turbulent simulations due to a wide spectrum of scales involved for such flows. For flows with eddy shocklets at high Reynolds numbers, the range of temporal and spatial scales widens significantly owing to the fact that the energy containing eddies have a much larger length scale than the dissipation structures. ${ }^{1}$ Local time-stepping algorithms allow larger time steps to be used for large flow structures away from the wall and in the quiet 
freestream regions. However, for the problems studied in this paper, TALTS is not required, because of the use of meshes with elements that are uniform in size. Thirdly, the CESE method has been shown to be able to capture waves and flow discontinuities with high fidelity. ${ }^{6,711-16}$ Finally, due to its space-time conservation formulation, the CESE method has been shown to allow easy and efficient implementation of non-reflecting boundary conditions. ${ }^{15-17}$ For turbulent flow simulations, substantial savings and ease of implementation can be achieved without the need for a buffer domain in the outflow/farfield regions. The genuinely multi-dimensional formulation of the CESE method, which is free of approximate Riemann solvers and dimensional splitting, offers a distinctly different way to simulate turbulent flows interacting with shocks.

This paper presents a brief description of the unstructured mesh algorithms and boundary conditions. Effects of boundary condition formulations on periodic boundaries and adiabatic viscous walls are also discussed. In preparation towards general compressible turbulent flow simulations, wave interaction with shocks is investigated for several benchmark-type problems, which includes acoustic wave/bow-shock, single vortex/normal-shock, and isotropic turbulence/normal-shock interactions. Numerical simulations of isotropic turbulent flow decay and the Taylor-Green vortex problem within a periodic box are performed using the $2^{\text {nd }}$ - and $4^{\text {th }}$ - order CESE schemes with isotropic tetrahedral meshes. Numerical computations of these problems are aimed at simulating more complex and realistic problems such as turbulent boundary layer-shock interactions over a compression corner and other benchmark cases. ${ }^{18}$

\section{Numerical Methods and Boundary Conditions}

This section briefly discusses the CESE formulation and the newly developed integral viscous boundary conditions.

\section{A. Numerical Formulation}

The space-time CESE method is formulated by using the integral form of the conservation laws. The space-time flux vector, $\vec{h}$, is defined as

$$
\vec{h}=\left(F_{x}, F_{y}, F_{z}, U\right)
$$

where flux components in three Cartesian coordinates $x, y$, and $z$ are written as $F_{x}, F_{y}$, and $F_{z}$, respectively. Vector $U$ represents the dependent conservative variables. The integral form of the governing equations can be written as

$$
\oint_{\Omega} \vec{h} \cdot d \vec{s}=\int_{V} G d V
$$

where the space-time flux vector is integrated over the surface, $\Omega$, of an arbitrary space-time domain, $V$. The space-time surface area vector is defined as $d \vec{s}=\vec{n} d A$ where $\vec{n}$ is the outward surface unit normal and $d A$ is the space-time surface area increment in $\Omega$. Notice that vectors, areas and volumes denoted here are generally defined in the space-time manifold, which could contain the temporal coordinate. The vector $G$ is associated with possible source terms such as body force, chemical reaction, or other external forcing. For three-dimensional compressible Navier-Stokes equations, the dependent variables are defined by $U=(\rho, \rho u, \rho v, \rho w, e)$ where $\rho, u, v, w$, and $e$ represents density, three velocity components, and total energy per unit volume $\left(e=\frac{p}{\gamma-1}+\frac{\rho}{2}\left(u^{2}+v^{2}+w^{2}\right)\right)$, respectively. Flux vectors $F_{x}, F_{y}$, and $F_{z}$, contain five elements to incorporate mass, three momentums, and energy conservation in the spatial coordinate $x, y$, and $z$, respectively. When the source vector, $G$, is zero and the governing equations reduce to

$$
\oint_{\Omega} \vec{h} \cdot d \vec{s}=0
$$

Equations (2) and (3) are quite general and many conservation laws can be cast in this form. The discretized form of Eq. (3) for a tetrahedral element can be expressed as

$$
\sum_{i=1}^{N} \sum_{k=1}^{4} \vec{h}_{i k} \cdot \Delta \vec{s}_{i k}=0
$$

In Eq. (4), the space-time flux conservation is summed over a total of $\mathrm{N}$ conservation elements and $k$ represents the indices of the neighboring elements. For a tetrahedral element, there are a total of four neighboring conservation elements and $k$ varies from $1-4$. The conservation elements $\Delta \vec{s}_{i k}$, are staggered 
with the solution elements where the dependent variables, $U_{i}$, vary according to the Taylor series expansion,

$$
U_{i}(x, y, z, t)=U_{0 i}+U_{t i}\left(t-t_{0}\right)+U_{x i}\left(x-x_{0}\right)+U_{y i}\left(y-y_{0}\right)+U_{z i}\left(z-z_{0}\right)
$$

where $\left(x_{0}, y_{0}, z_{0}, t_{0}\right)$ is the solution point within the solution element $i$ defined as the centroid of all surrounding conservation elements. For high-order CESE schemes, Eq. (5) would contain higher (second and third for fourth-order schemes) derivatives in the Taylor series expansion. Equations (3)-(5) constitutes the baseline non-dissipative scheme. However, for non-linear equations, proper numerical dissipation has to be added to the non-dissipative baseline scheme, for maintaining numerical stability. See Refs. 4, 6 and 7 for more details regarding how numerical dissipation can be introduced properly under the CESE framework.

Two distinct properties are implied in Eq. (4). Firstly, since the conservation elements share common faces with each neighbor and the interface flux is a direct function (without any reconstruction or Riemann approximation) of dependent variable $U$ defined in Eq. (5), integration (summation) with the same orientation implies that the fluxes cancel each other except near the domain boundaries. Thus, total mass, momentum, and energy fluxes are conservative in each discretized element as well as the entire domain. Therefore, a derived flow quantity such as entropy possesses the same degree of local and global conservation for isentropic flows. In fact, for inviscid flows, the only source of total entropy change would come from the boundaries according to Eq. (4). Secondly, the discretized conservation equation (4) is in a strong space-time flux form and conservation of fundamental laws is evaluated without invoking any concept of weak solution. For flow equations, the fact that each conservation element has faces only in the smoothly defined region covered by the corresponding solution element forms the basis for a consistent and genuine multi-dimensional scheme. Such formulations in the integral form are valid without any approximation or reconstruction even for flows with shocks owing to the fact that fundamental laws still apply across flow discontinuities. This property is in contrast to most CFD methods formulated either by finite difference approximation of the differential equations or by formulations to make the flux differential unique across the discontinuous cell interfaces. These unique properties of the CESE method in particular make it an appropriate method to resolve waves and flow discontinuities synergistically.

Proper boundary conditions for Eq. (4) are of integral form. The flux integration requires the knowledge of all flux vectors along the boundary. At any boundary face, all flux vectors are either specified (for inflow) or extrapolated (for non-reflecting conditions) with the exception of inviscid or viscous walls. Wall boundary conditions are relatively easy to implement in the integral form. No ghost points are used in the wall boundaries and the conservation element degenerates into a triangle in 2D and a tetrahedron in 3D. For these wall boundaries, the mass flux is zero in the absence of mass influx from the wall. The only non-zero flux comes from wall pressure for either inviscid/adiabatic wall or from heat flux if the wall temperature is specified,

$$
(\vec{h} \cdot \Delta \vec{s})_{w}=\left(0, p_{w a} n_{x}, p_{w a} n_{y}, p_{w a} n_{z}, k \partial T / \partial n\right) \Delta A
$$

where $p_{w a}$ is the wall pressure, $\left(n_{x}, n_{y}, n_{z}\right)$ is the surface unit outward normal at the boundary element, $k \partial T / \partial n$ is the surface heat flux, and $\Delta A$ is the surface area of the boundary element. For second-order accurate schemes, both $p_{w a}$ and $k \partial T / \partial n$ are evaluated according to the adjacent interior solution element at the face center; and for high-order schemes, they can be evaluated using high-order quadrature along the entire boundary face. The normal temperature gradient $\partial T / \partial n$ is evaluated using the Taylor series expansion within the adjacent interior element along with the projection of $\nabla T$ in the surface normal direction.

In contrast to common CFD algorithms, in which the temporal derivative is treated separately by using finite differences and only spatial derivatives are integrated via either finite volume or Galerkin methods, the CESE method integrates the conservation laws consistently over the entire discretized spacetime domain. Such a synergistic formulation offers uniform temporal and spatial solution accuracy up to the designed order. ${ }^{6,7}$ For flow simulations, the conservation in both space and time has the potential to improve the temporal accuracy. In the CESE method, the solution $U$ is assumed to vary according to Taylor series expansion around the solution element. Numerical integration of Eq. (2) or (3) is done by conservation elements that have faces only through valid regions of the solution elements. No numerical artifacts or flux reconstruction are needed in the numerical integration. As a result, the solution accuracy depends only on the Taylor series expansion in the space-time domain. Details of the numerical formulation used here can be found in Refs. 4, 5 and 7. 


\section{B. Validation of Integral Viscous Wall Boundary Treatment}

To test the viscous boundary treatment, a Mach 3 flow over a flat plate boundary layer was computed. This same case has been investigated in Ref. 7 by using a $41 \times 21$ uniform structured mesh that was sliced into triangles. High-order solutions with this mesh compared well with the compressible boundary layer similarity solutions at $\operatorname{Re}=10^{5}$. To test the performance for high aspect ratio elements, $41 \times 31$ and $41 \times 51$ meshes with different geometric stretching that results in the near wall aspect ratio of 50 and 350 , respectively, were tested. The computed velocity profiles using the $4^{\text {th }}$-order CESE method are compared with boundary-layer solutions in Fig. 1. Results from both meshes agree quite well with the boundary-layer solutions near the wall. Around the shoulder of the velocity profiles, there are some discrepancies. This is to be expected because of poor resolution in that region due to strong mesh stretching.

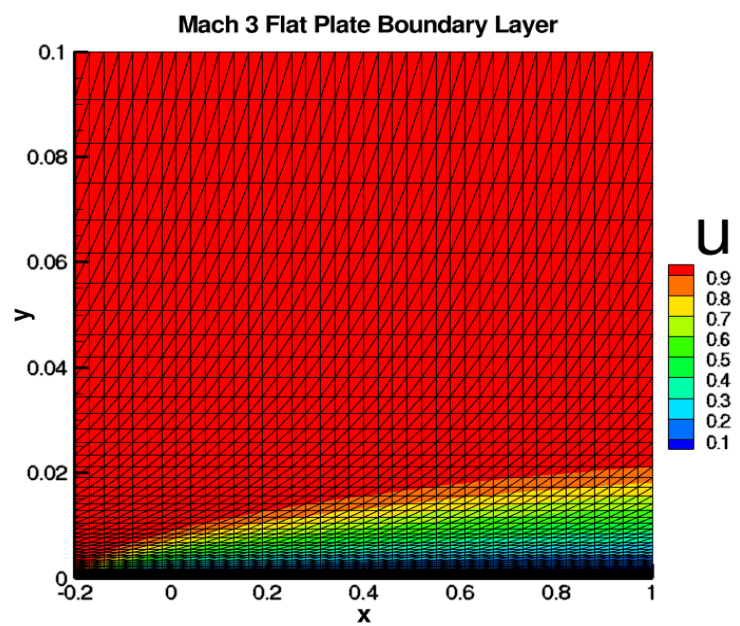

(a)

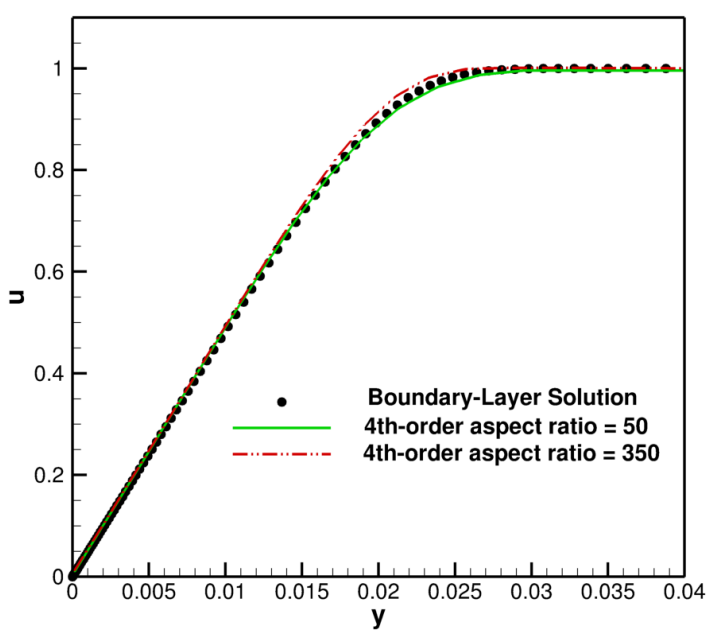

(b)

Figure 1. Streamwise velocity contours and profiles compared with similarity boundary layer solutions for a Mach 6 flat-plate boundary layer, both $x$ and $y$ coordinates are in meters (a) $u$ velocity contour and mesh (b) $u$ profiles versus wall-normal coordinate at $x=0.8 \mathrm{~m}$.

For the flow simulation of isotropic turbulence decay, to be discussed later, a cubic domain with a side length of $2 \pi$ is used. Isotropic tetrahedral elements are generated inside the box domain and periodic boundary conditions are enforced at all the six faces. There are at least two ways to implement periodic boundary conditions for tetrahedral meshes. In the first approach, an image element is constructed at all periodic boundaries. The solution at the ghost point is matched to its periodic neighbor located on the opposite side of the domain at each time step. The main advantage of this approach is that boundary faces at the periodic boundaries need not to be perfectly matched. However, there are numerical errors associated with the mismatched periodic meshes due to interpolation and geometry mismatch. The second approach copies both solutions and geometries of the matched neighboring periodic element to the present periodic boundaries. Numerical computations are done at periodic faces as if they are interior neighbors. The mesh is perfectly continuous across the periodic boundary. For parallel computations, however, this approach would slightly increase the communication overhead. For waves or small-scale oscillations propagating across the periodic boundaries, the second approach is to be preferred.

\section{Results}

The second- and high-order CESE methods, in conjunction with the boundary conditions discussed are to be used for turbulent flow simulations with tetrahedral meshes. The motivation is to assess the overall simulation accuracy and effects of orientation-free meshes in capturing small-scale turbulent flow features. Order of accuracy for flows with discontinuities for the CESE method has been established in detail in Ref. 6. High-order accuracy has also been established for the linear advection equation, linearized and non-linear Euler equations. ${ }^{719-20}$ In this article, interactions of waves, vortex, or isotropic 
turbulence with normal or bow shocks are studied using unstructured triangular or tetrahedral meshes to assess the performance of the space-time conservative CESE schemes. In addition, numerical computations are also performed for three-dimensional decaying isotropic turbulence (with a high turbulent Mach number) and the inviscid Taylor-Green vortex. ${ }^{32}$ A CFL number of 0.7-0.9 was typically used to carry out the numerical computations presented in this paper. All of the above-mentioned flow problems represent various aspects that need to be assessed before CESE schemes can be applied to simulate the more complex shock/turbulent boundary layer interaction problem.

For the speed regimes from transonic and up, unsteady wave interactions with flow discontinuities see their importance in countless applications. Unfortunately, special numerical treatments to stabilize solution across the shocks are needed for most numerical schemes. Large numerical dissipation, if used around flow discontinuities, can have a significant impact on the solution accuracy. For this reason, the interaction of unsteady waves (in the form of acoustic, vorticity, or entropy waves) with shocks has attracted attention from computational fluid dynamics researchers for decades. Several one- or twodimensional benchmark problems exist but none have exact solutions for comparison. The common practice is to use a "numerically exact" solution obtained using a very refined mesh for validation. For small perturbations across the shock, linear theory ${ }^{21,22}$ does exist. In such analysis, the pre- and post-shock perturbations expressed as acoustic, entropy, or vorticity waves are related according to the jump conditions. In general, small perturbations across the shock are governed by a set of perturbed RankineHugoniot differential equations ${ }^{23}$ that can be solved numerically with some knowledge of the initial waveform of the shock oscillations. Unfortunately, these theoretical or numerical solutions can only provide a means to validate the ratio of gross measures of perturbations across the shock, such as the integrated vorticity or the turbulent kinetic energy. For some of the benchmark problems involving simple geometries, flow field details of waves/shock interactions have been obtained via stable and minimally damped numerical schemes. The state-of-the-art available includes compact-differencing schemes with high-order filters, ${ }^{24}$ weighted essentially non-oscillatory (WENO) methods, ${ }^{25}$ the space-time CESE method, ${ }^{4,5}$ and some other variations of the above-mentioned methods. In the discussion below, results obtained from the present approach with a compact domain are compared with those from these investigations. Most of these solutions in literature, with the exception of the CESE method, have been obtained using a hybrid treatment by using the upwind-based WENO operator to stabilize flow discontinuities and switching to low-dissipation schemes away from the shock to resolve the unsteady waves. In the following sections, several wave/shock interaction problems are investigated using the CESE method that is free of hybrid treatment with unstructured triangular or tetrahedral meshes.

\section{A. Acoustic Wave and Bow-shock Interaction}

Interactions between acoustic waves and a bow shock occur in many supersonic or hypersonic blunt body configurations. Freestream perturbations in the form of acoustic waves propagate across the leading-edge bow shock, generating disturbances that impinge on the blunt body surface, and affecting the surface pressure and generating boundary-layer instability waves. This problem is of great importance and appears to be more challenging than its $1 \mathrm{D}$ counterpart. The resolution requirement of acoustic waves could incur numerical instability near the stagnation region of the bow shock known as the carbuncle phenomenon. ${ }^{26,}{ }^{27}$ Some solutions with limited grid resolution are available at Mach 10 from previous studies. ${ }^{28}$ Quasi-steady state solutions of a planar acoustic wave passing through a bow-shock, generated by a hypersonic flow over a sphere were computed as part of this study. The solutions were obtained by solving the Euler equations, using the $2^{\text {nd }}$-order scheme with triangular elements obtained by slicing a uniformly-spaced quadrilateral mesh. Three levels of meshes were used: $513 \times 193,1025 \times 385$, and $2049 \times 769$. For this acoustic wave problem and the subsequent vortex-shock interaction case, there exists a preferred wave/flow direction. Therefore, an isotropic triangular mesh may not provide significant advantages over the sliced triangular mesh used here.

The steady-state solutions obtained for a given Mach condition are superimposed with the fastmode (with a phase velocity of $1+1 / M$ ) planar acoustic waves at $50 \mathrm{kHz}$ propagating downstream to interact with the bow shocks. The amplitude of the pressure disturbance (normalized by the pre-shock freestream pressure) is set at $10^{-4}$ to study linear propagation. In Fig. 2, the Fourier-transformed pressure perturbations obtained by the medium and fine mesh were compared for a freestream Mach number of 7.32. The results indicate that the solutions converged for the two finer meshes. The solutions presented hereafter were obtained by using the medium mesh. 


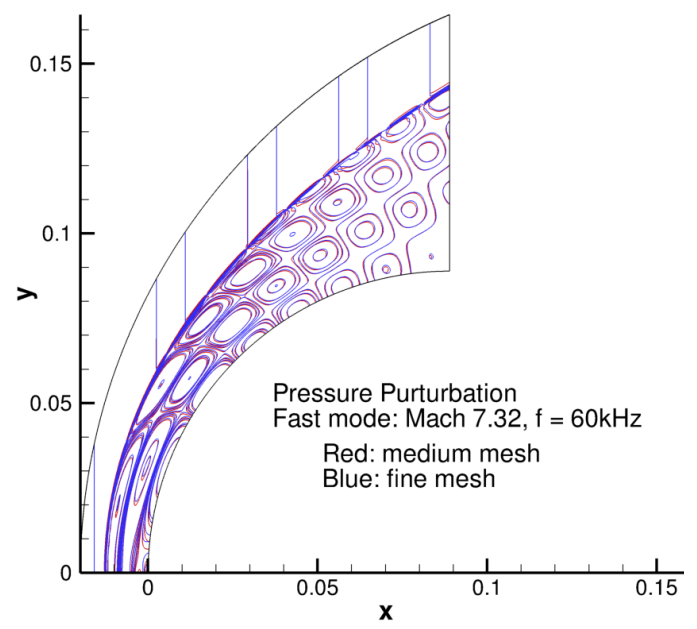

Figure 2. Acoustic wave/bow shock interaction for a Mach 7.3 flow past a hemi-sphere. Fourier transformed perturbation solutions for pressure are shown for the medium and fine mesh.

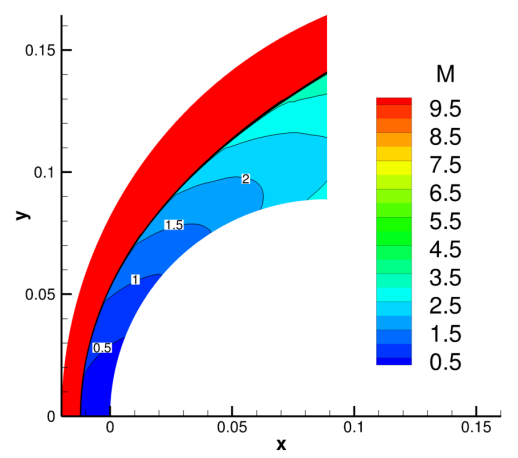

(a)

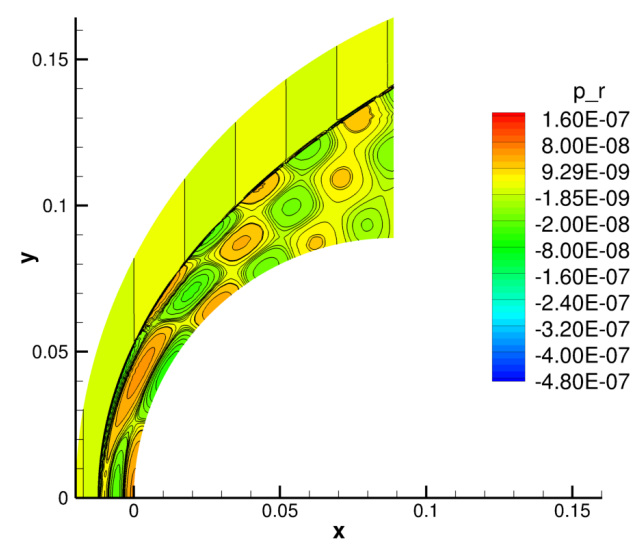

(b)

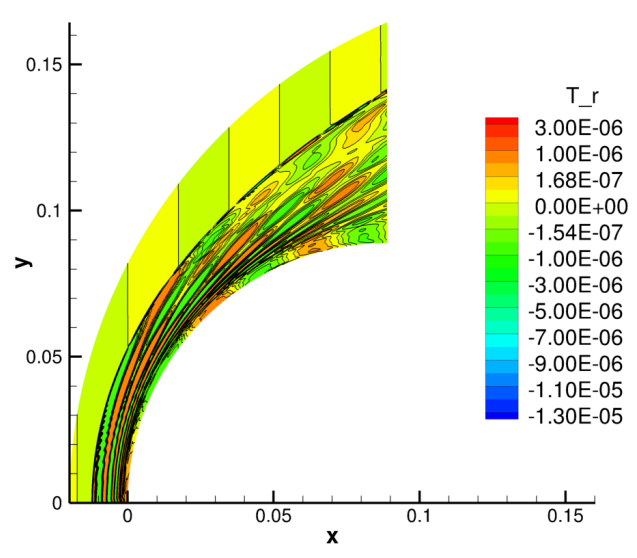

(c)

Figure 3. Acoustic wave/bow shock interaction for a Mach 10 flow past a hemi-sphere: (a) steadystate Mach contours; (b) Fourier transformed perturbation solutions for pressure; (c) Fourier transformed perturbation solutions for temperature.

The Fourier transformed perturbation solutions for the Mach 10.0 condition are shown in Figs. $3(b)$ and 3(c) for pressure (normalized by pre-shock freestream density multiplied by the square of the velocity) and temperature (normalized by pre-shock freestream temperature), respectively. Interestingly, the post-shock solutions exhibit quite different scales for the pressure and the temperature perturbations, 
respectively. Post-shock temperature perturbations have a shorter length scale and larger amplitude than the other flow quantities. All small perturbations are resolved without numerical difficulties for the strong bow shock case presented in this case. The slow-mode solutions (with a phase velocity of $1-1 / M$ ) show similar patterns to that of the fast-mode shown here, with the exception that the perturbation structures lean more towards the bow shock. The effects of these rather complex post-shock patterns on boundary-layer receptivity for blunt bodes are under investigation and will not be discussed here. This example demonstrates the effectiveness of the current numerical scheme in computing acoustic wave interacting with a strong hypersonic bow shock.

\section{B. Isentropic Vortex and Normal Shock Interaction at Low Supersonic Speed}

Turbulent flows consist of vortical structures with a broad range of scales. Interaction of a single vortex with a strong shock is of fundamental interest for numerical computations. Many researchers have studied an isentropic vortex propagating through a normal shock to assess the accuracy of their schemes, as well as to explore the details of the flow physics. ${ }^{29,30}$ In these investigations, the rectangular domain consists of two uniform-flow regions to represent the upstream and downstream conditions of a normal shock. The upstream and downstream Mach numbers $M_{1}$ and $M_{2}$, respectively, can be found from the normal shock table. Typically, the domain size is large enough to not let the truncated boundaries interfere with the vortex propagation. A substantial buffer domain at the downstream boundary is needed to avoid spurious wave reflections. In Ref. 29, the grid is clustered near the shock and gradually stretched away from it to hold the shock in the right position; and to improve the solution accuracy across the shock, a high-order compact differencing scheme is used. In Ref. 30, a $4^{\text {th }}$-order WENO scheme is used in conjunction with non-reflecting boundary conditions and no grid clustering near the shock is needed.

The first run was carried out with $M_{1}=1.29$ (thus, $M_{2}=0.79$ ), with a core vortex diameter of 0.6 . The computational domain extends from 0 to $2 \pi$ for both directions. A $256 \times 256$ quadrilateral mesh is sliced to form about 131,000 triangular elements. Initial conditions from Refs. 29 and 30 are used in the following calculations. A single vortex is superimposed at the upstream region centered at $x=\pi / 2$ and $y=\pi$. The domain size is relatively small compared to other investigations. ${ }^{29,30}$ The subsonic boundary conditions corresponding to the post-shock Mach number, $M_{2}$, are imposed at the right boundary; and the normal shock conditions associated with the prescribed upstream and downstream Mach numbers are imposed at the top and bottom boundaries, respectively. Periodic conditions can also be used but are not required. Figure 4(a) compares the solutions obtained by imposing periodic as well as jump boundary conditions at the top and bottom boundaries. The use of the fixed jump conditions and subsonic outflow are stable and do not incur spurious wave reflections, as shown in the figure. The jump condition produces additional waves at both the shock corners, but appear not to affect the major vortex structures. Furthermore, the motivation behind the use of a computational setup that involves a relatively coarse mesh and the close proximity of farfield/outflow boundary locations, are to test the numerical stability and solution quality of the CESE schemes under such sub-optimal conditions. No buffer domains are required. Figure 4(b) compares solutions obtained by the $2^{\text {nd }}$ - and $4^{\text {th }}$-order schemes. The results show very small difference in the major vortical structures and the radiated acoustic waves for the present mesh. 


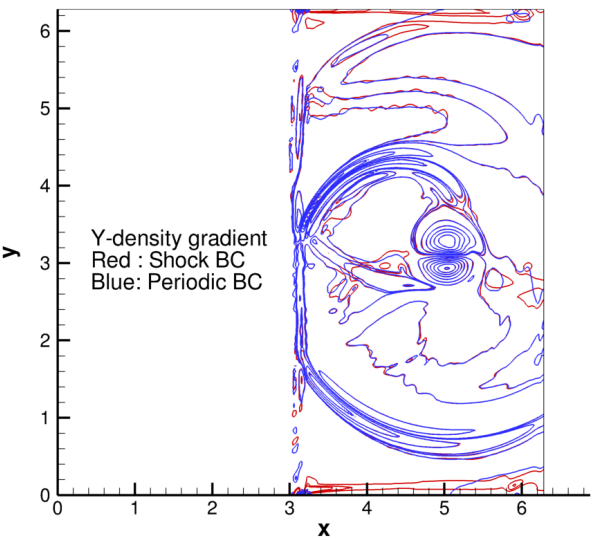

(a)

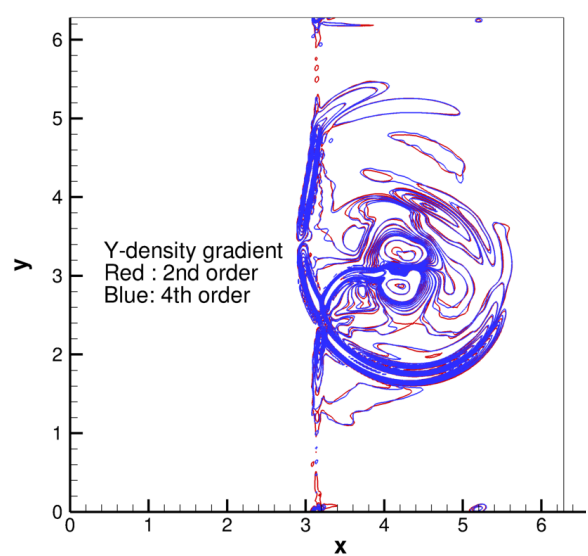

(b)

Figure 4. Interaction of a single vortex with a normal shock with an upstream Mach number of 1.29, showing the density gradient contours obtained: (a) two different boundary conditions at the top and bottom boundaries; (b) using the $2^{\text {nd }}$ - order and $4^{\text {th }}$-order accurate schemes.

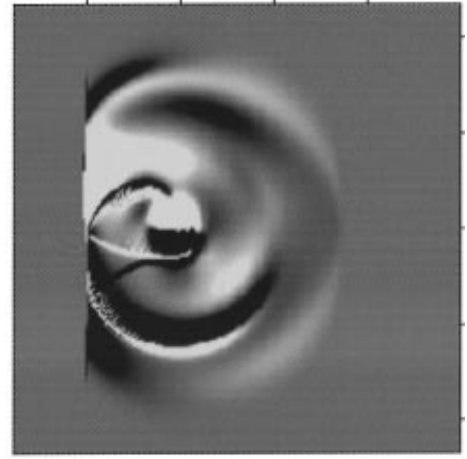

(a)

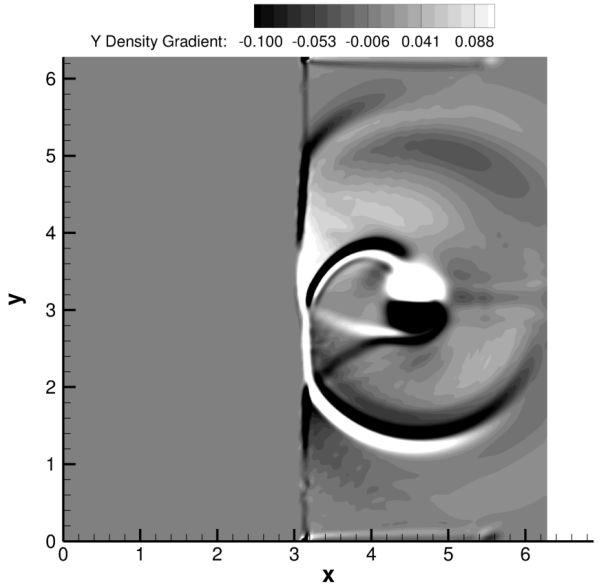

(c)

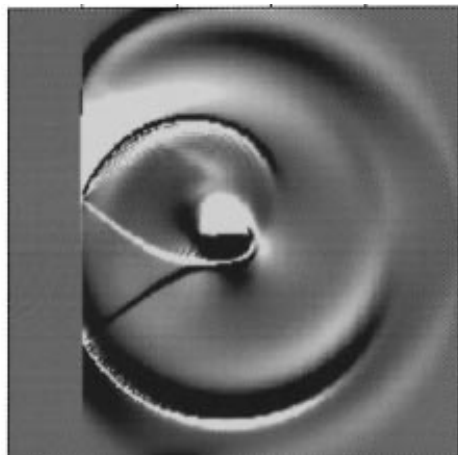

(b)

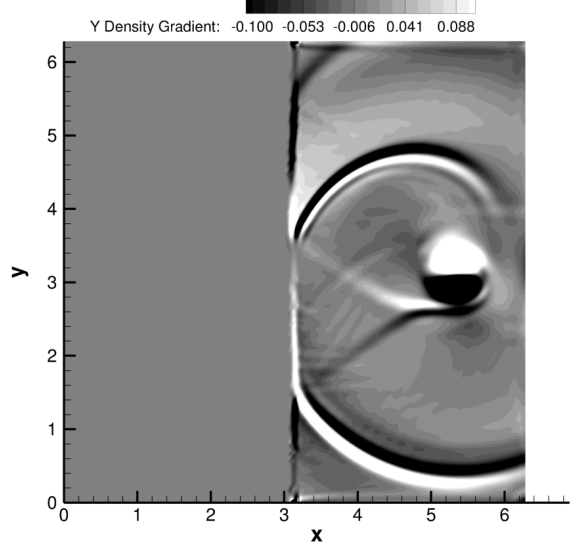

(d)

Figure 5. Interaction of a single vortex with a normal shock with an upstream Mach number of 1.29, showing numerical Schlieren contours for normal density gradient: (a) rotated image of Fig. 12(a) from Ref. 29; (b) rotated image of Fig. 12(b) from Ref. 29; (c) computed solutions corresponding to (a); (d) computed solutions corresponding to (b). 
Figure 5 shows the comparison of present results with those from Inoue and Hattori ${ }^{29}$ for a vortex Mach number (based on the tangential velocity of the vortex) of 0.39 . The qualitative agreement between the two solutions is good. The fixed shock conditions at the top and bottom boundaries produce perturbations that are confined near the shock corners. The downstream non-reflecting boundary conditions yield minimal boundary reflections. The leading acoustic waves, the subsequent vortex cores, and the accompanying flow structures, all exit the domain without producing undesirable spurious waves that can propagate upstream, even when the vortex core approaches the downstream boundaries as shown in Fig. 5(d). To visualize the acoustic wave generated by the vortex/shock interaction, the downstream pressure ratio distribution $\left(d p=\left(p-p_{2}\right) / p_{1}\right.$, where $p$ is the local pressure, and $p_{1}$ and $p_{2}$ are the pre- and post-shock static pressure, respectively) is computed and compared to the results from Ref. 30 in Fig. 6. The quadruple acoustic field generated by the vortex/shock interaction is captured by the present method and qualitatively compares well with the solution from Ref. 30. No noticeable acoustic wave reflections at the boundaries are evident. When the vortex penetrates through the shock, both acoustic and entropy waves are generated. Figure 7(a) shows the $z$-vorticity (normalized by the pre-shock freestream conditions) contours at a slightly later time than that in Fig. 6. The vortex core is now very close the boundary, the circular core structure has been squeezed and rotated after passing through the shock. The rim of the vortex also develops into a larger, counter rotating (negative $z$-vorticity) structure with a pair of lambda-shaped legs. Interestingly, despite very minimal rotation of the vortex core after passing through the shock, the entropy perturbations develop into a spiral structure downstream of the shock as shown in Fig. 7(b). There is also a wedge of strong entropy generation confined between the lambda vortex legs and the normal shock. In general, the vortex weakens after the shock by passing energy to the entropy and acoustic perturbations.

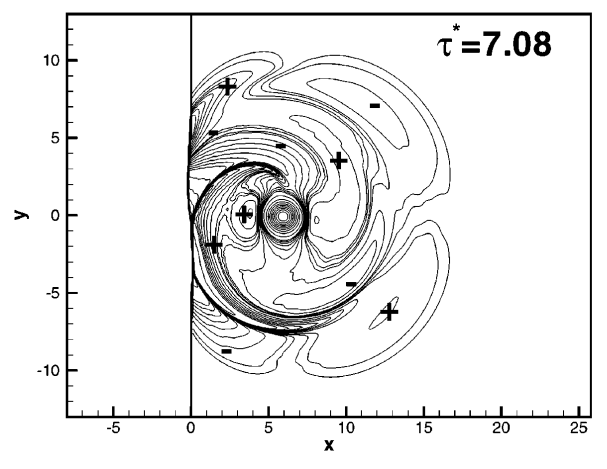

(a)

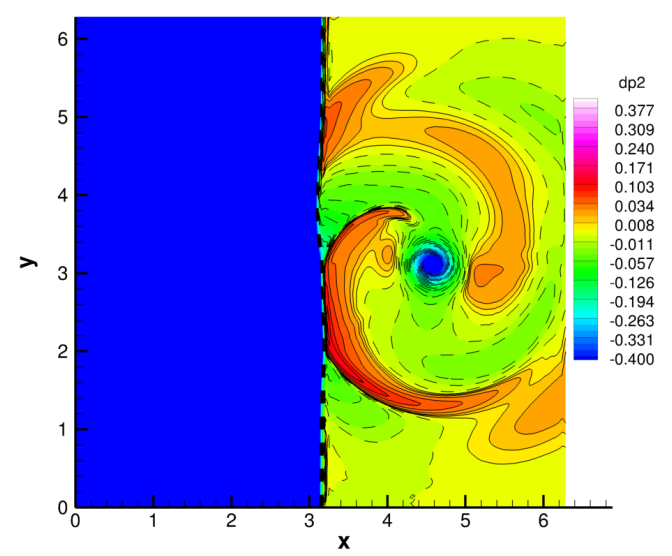

(b)

Figure 6. Comparison of computed acoustic pressure field with that from Fig. 12(b) of Ref. 30 at Mach 1.2 and a vortex Mach number of 0.4: (a) Results of Grasso and Pirozzoli; ${ }^{30}$ (b) Present results.

In both Refs. 29 and 30, the authors indicate that the wind-tunnel Schlieren photos from the experiment of Dosanjh and Weeks ${ }^{31}$ show more rotating motion as the vortex passes through the shock under similar shock conditions. Several legs spin off the core vortex due to rotation and there are no apparent lambda-shape legs observed in the experiment. The reason for the difference is still unknown. To investigate how the strength of the shock could affect the vortical structure development downstream, a new set of calculations were performed for $M_{1}=2$ and $M_{2}=0.58$. The vortex Mach number is set to be 0.8. The results are shown in Fig. 8. At this stronger shock condition and relatively large vortex speed, the normal shock remains intact (see discussion below for broken-shock situations taking place when isotropic turbulence structures interact with normal shocks). The resulting downstream vortical structure shows much stronger spiral motion with several legs developing as can been seen in the figure. Interestingly, the post-shock vortical structure shows a tornado-like shape and still with a pair of lambda-shape legs. The results clearly indicate that the rotating motion of the vortex core strongly depends on the shock conditions and the vortex speed. 


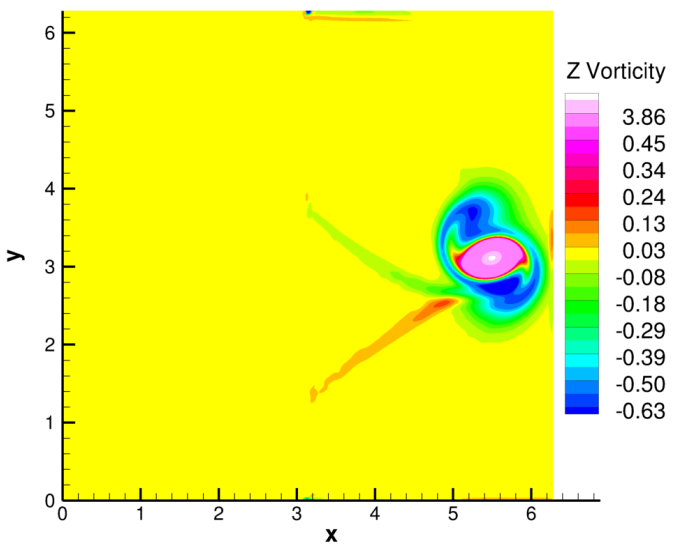

(a)

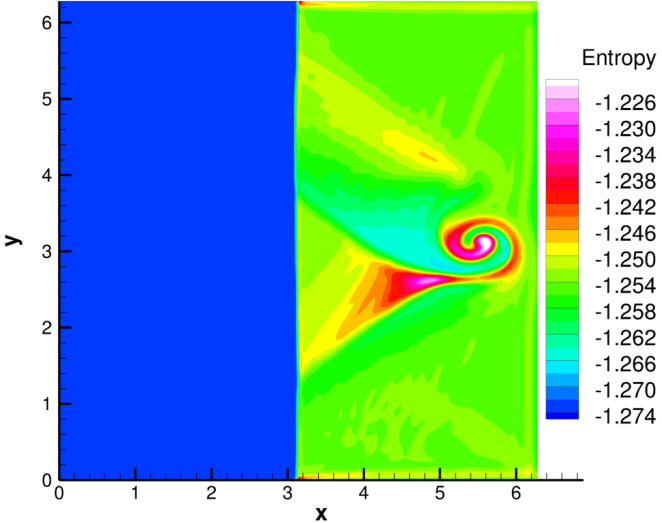

(b)

Figure 7. Post-shock contours for $M_{1}=1.2$ and a vortex Mach number of 0.4 : (a) $z$-vorticity; (b) entropy (both quantities normalized by pre-shock conditions).

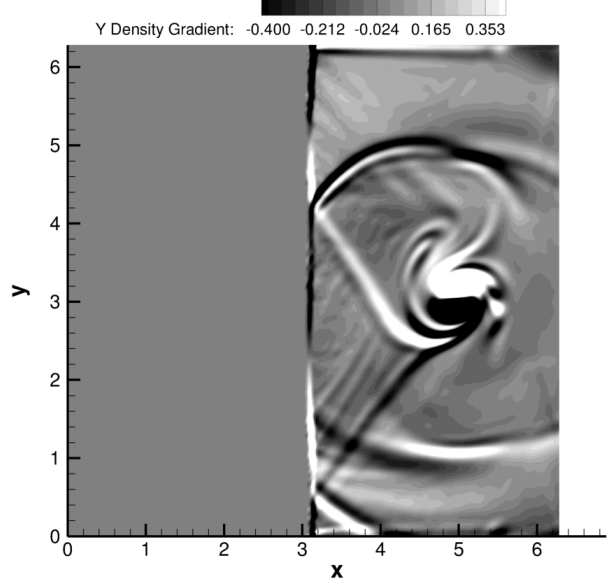

(a)

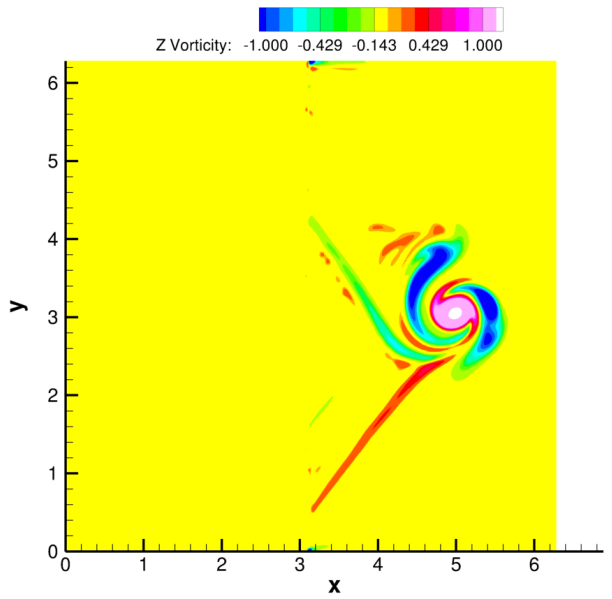

(b)

Figure 8. Post-shock solutions for strong interaction at $M_{1}=2.0$ and a vortex Mach number of 0.8 : (a) numerical Schlieren (y density gradient); (b) $z$-vorticity (normalized by pre-shock conditions).

\section{Inviscid Taylor-Green Vortex Problem}

The inviscid Taylor-Green vortex problem ${ }^{32}$ concerns time evolution of several incompressible vortices inside a periodic box. The initial conditions of these vortices give rise to flow instability that leads to breakdown of the initial vortices into small-scale flow structures and eventually to full turbulence. The breakdown starts happening after an initial period of three non-dimensional units, when the vortices begin to stretch and interact with adjacent vortices. Eventually, the turbulent flow decays. Given the inviscid nature of this problem, thereby free of viscous dissipation, it is a good test for assessing the numerical dissipation properties of a numerical algorithm and simulation codes, as well as their ability to simulate the breakdown of the vortices into turbulence with high fidelity.

The computational domain of this problem is a cubic box with an edge length of $2 \pi$. Periodic boundary conditions are enforced in all three directions. The meshes used for these simulations predominantly contained isotropic tetrahedrons that were generated using commercial grid generation tools. To facilitate comparison with other computations in literature, the mesh size noted here indicates its equivalent structured mesh size. For instance, a $64^{3}$ tetrahedral mesh implies that the total number of isotropic tetrahedrons is roughly $64^{3}$. A sequence of meshes with sizes of $64^{3}, 122^{3}$, and $192^{3}$ have been used for both $2^{\text {nd }}$ - and $4^{\text {th }}$-order schemes. 
The Euler results are compared with the semi-analytical results of Brachet et al. ${ }^{33}$ Figure 9 shows the initial vortex pattern and the flow structure at two later times, when the breakdown of the vortex starts and its subsequent evolution into smaller scales. In Fig. 9, Q-criterion ${ }^{34}$ is used to identify and represent the vortex cores. The breakdown to small-scale, turbulent like flow structures is evident. The slightly rough surface textures on these isosurfaces are due to the Tecplot ${ }^{\mathbb{B}}$ interpolation on a tetrahedral mesh. The computed total kinetic energy and enstrophy are compared with Brachet et al. solutions ${ }^{33}$ in Fig. 10, and the agreement is good up to four non-dimensional time units. Beyond this time, as rapid build-up of fine-scale structures ensues, numerical dissipation of the algorithm begins to dissipate the kinetic energy. An accurate numerical method for this inviscid problem should preserve the kinetic energy, as required by the Euler equations in the near-incompressible limit, while allowing enstrophy (flow rotation) to grow rapidly during the vortex breakdown to turbulence stage. Furthermore, it should not suffer from any numerical stability because of the presence of severely under-resolved features. As seen from Fig. 10 (a) and (b), although the kinetic energy decreases, the enstrophy levels continue to rapidly increase in the initial stage, with increase in mesh resolution and/or order of accuracy and there is no evidence of any numerical instability. A special form of discretization that preserves kinetic energy, ${ }^{35}$ in the incompressible limit, has been able to preserve kinetic energy for a much longer duration. Such formulation was required to maintain numerical stability. Kinetic energy preservation alone cannot be used as an indicator of the performance of a numerical scheme for this particular problem, ${ }^{36}$ as one also needs to take into account the prediction of the enstrophy growth, at least up to a duration when the scales in the flow are still well-resolved. The results obtained by CESE are comparable to those from some of the state-of-the-art methods used in DNS of turbulent flows, ${ }^{35}$ as seen by the metrics provided in Table 1. The maximum enstrophy levels shown in Fig. 10(b) are substantially higher than the viscous benchmark solutions provided in the high-order benchmark problems. ${ }^{18}$ If the numerical dissipation was further reduced in the $4^{\text {th }}$-order simulations, the computed enstrophy values followed the Brachet et al. solution ${ }^{33}$ and continued to increase, until it quickly became unstable due to the lack of numerical dissipation. As pointed out by Brachet et al., ${ }^{33}$ this is to be expected, as there exists a finite-time singularity at $t>4.0$. The present inviscid results show that with added numerical dissipation, the results appear to be the limit of higher Reynolds number solutions, showing regions of vortex breakdown and decay. Possible causes are improper numerical dissipation control for the low Mach number regions (in the order of $10^{-3}$ ) present in the flow field or the lack of low Mach number pre-conditioners for compressible formulations. The former may also explain why the kinetic energy shown in Fig. 10(a) is not preserved perfectly when the vortices begin to breakdown into smaller scales.

Table 1. Accuracy metrics for the inviscid Taylor-Green vortex simulation.

\begin{tabular}{|c|c|c|c|c|c|c|c|c|c|}
\hline & \multirow{2}{*}{$\begin{array}{l}\text { Brachet } \\
\text { et al. }^{33}\end{array}$} & \multirow[b]{2}{*}{ Hybrid $^{35}$} & \multirow[b]{2}{*}{ WENO $^{35}$} & \multicolumn{3}{|c|}{ CESE $2^{\text {nd }}-$ Order } & \multicolumn{3}{|c|}{ CESE $4^{\text {th }}$-order } \\
\hline & & & & $\begin{array}{l}64^{\wedge} 3 \\
\text { mesh }\end{array}$ & $\begin{array}{l}122^{\wedge} 3 \\
\text { mesh }\end{array}$ & $\begin{array}{l}196^{\wedge} 3 \\
\text { mesh }\end{array}$ & $\begin{array}{l}64^{\wedge} 3 \\
\text { mesh }\end{array}$ & $\begin{array}{l}122^{\wedge} 3 \\
\text { mesh }\end{array}$ & $\begin{array}{l}196^{\wedge} 3 \\
\text { mesh }\end{array}$ \\
\hline $\begin{array}{l}\text { Kinetic } \\
\text { energy at } \\
t=5\end{array}$ & 1.00 & 1.00 & 0.916 & 0.896 & 0.969 & 0.979 & 0.901 & 0.974 & 0.980 \\
\hline $\begin{array}{l}\text { Enstrophy } \\
\text { at } t=3.5\end{array}$ & 3.46 & 3.33 & 3.13 & 2.73 & 3.24 & 3.38 & 3.24 & 3.26 & 3.29 \\
\hline
\end{tabular}




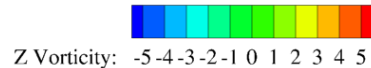

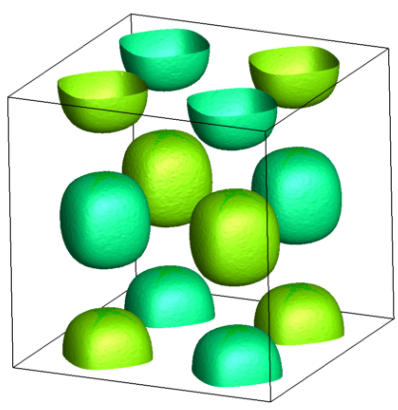

$\mathrm{Zg}^{\mathrm{Y}}$

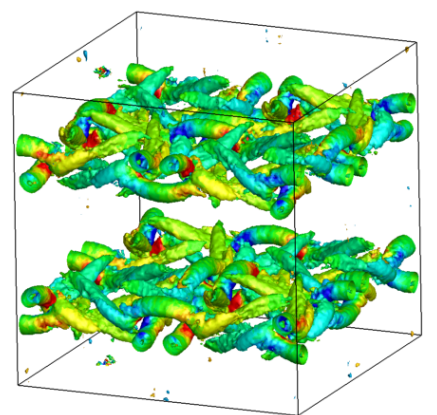

Time $=5.38$

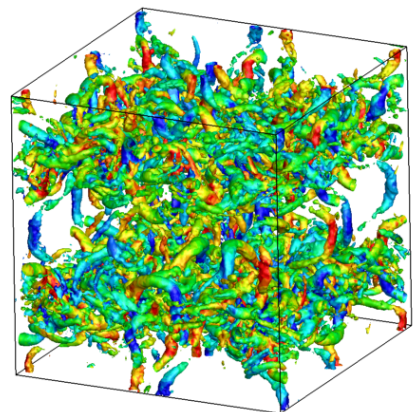

Time $=10.8$

Figure 9. Computed isosurfaces of $Q$-criterion shaded with $z$-vorticity (normalized by the reference conditions at Mach 0.1) at three different times.

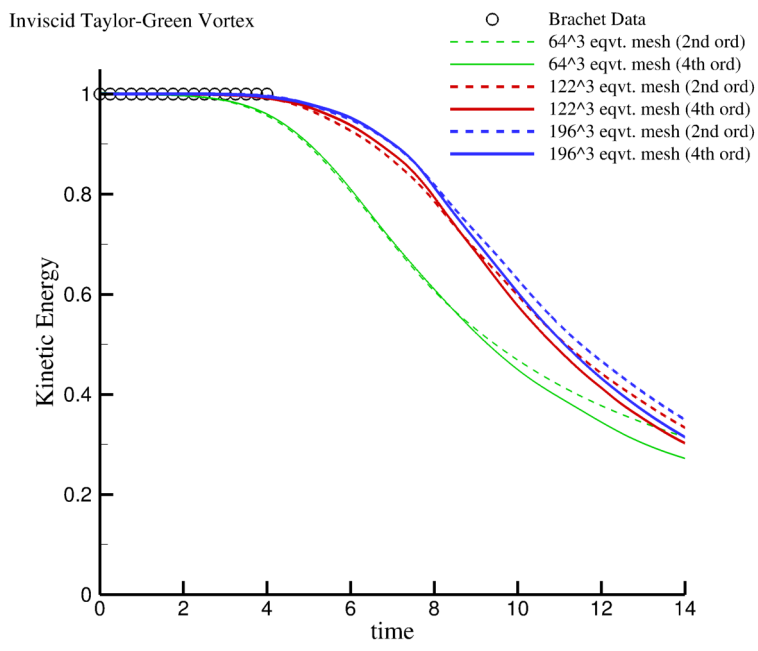

(a)

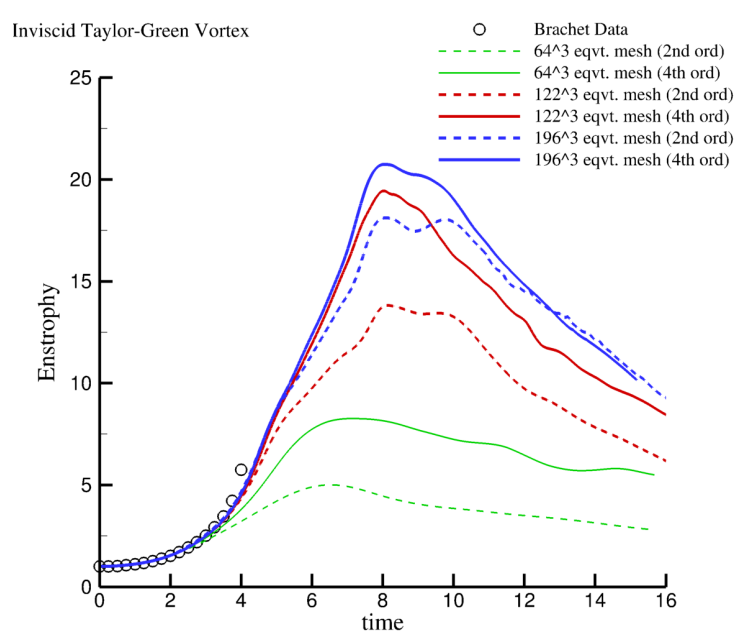

(b)

Figure 10. Time evolution of normalized total kinetic energy and enstrophy (by initial values) computed for the Taylor-Green vortex problem compared with semi-analytical results from Brachet et al. ${ }^{33}$ : (a) kinetic energy; (b) enstrophy.

\section{Decay of Homogeneous Isotropic Turbulence}

Decay of homogeneous isotropic turbulence in a box has been a standard test case for direct numerical simulation algorithms and computer codes (e.g., Ref. 37). In these simulations, threedimensional Navier-Stokes equations are solved on a cubic box with an edge length of $2 \pi$ by applying periodic boundary conditions in all three directions. The box is initialized with a theoretical isotropic turbulent flow by using the procedure described in Ref. 38. Two important non-dimensional parameters characterizing the state of the flow are (i) turbulent Mach number, $M_{t}$, and (ii) Reynolds number based on the Taylor microscale, $R e_{\lambda}$. They are defined as follows:

where,

$$
M_{t}=\frac{\sqrt{\left\langle u_{i} u_{i}\right\rangle}}{\langle c\rangle} ; R e_{\lambda}=\frac{\langle\rho\rangle u_{r m s} \lambda}{\langle\mu\rangle}
$$




$$
\boldsymbol{u}_{r m s}=\sqrt{\frac{\left\langle u_{i} u_{i}\right\rangle}{3}} ; \lambda=\frac{\left\langle u_{1}^{2}\right\rangle}{\left\langle\left(\partial u_{1} / \partial x\right)^{2}\right\rangle}
$$

In the above equations, the symbol \langle\rangle stands for ensemble average and $\lambda$ represents the Taylor microscale. The flow field is initialized by setting up a random velocity field, $u_{i, 0}$, that is solenoidal and also satisfies a chosen energy spectrum and the initial turbulent Mach number:

$$
E(k) \sim k^{4} \exp \left(-2\left(k / k_{0}\right)^{2}\right) ; \frac{3 \boldsymbol{u}_{r m s, 0}^{2}}{2}=\frac{\left\langle u_{i, 0} u_{i, 0}\right\rangle}{2}=\int_{0}^{\infty} E(k) d k
$$

where, $k$ stands for the wavenumber magnitude. $k_{0}$, the most energetic wavenumber, is taken as 4 . For the chosen energy spectrum, the initial Taylor length scale, $\lambda_{0}$, is $2 / k_{0}$. The initial density and pressure fields are assumed to be constant and the remaining parameters are set based on $M_{t, 0}$ and $R e_{\lambda, 0}$. There are alternate approaches of initializing the fluctuations in density and pressure fields to make it more consistent; ${ }^{39}$ but those will not be discussed here.

Similar to Ref. 1, most simulations for isotropic turbulence have been performed using structured or unstructured hexahedral meshes. This paper focuses on simulations done using isotropic tetrahedrons that have been generated using commercial grid generation tools. The computational domain, boundary conditions, and the mesh resolutions used for these simulations were the same ones as those used for the Taylor-Green vortex problem. Meshes with sizes of $64^{3}, 122^{3}$, and $192^{3}$ have been used for both the $2^{\text {nd }}$ and $4^{\text {th }}$-order accurate schemes. Results from $122^{3}$ mesh computed by the $4^{\text {th }}$-order schemes are shown below in Figs. 11-13. In general, $2^{\text {nd }}$-order solutions with the $192^{3}$ mesh give nearly identical large-scale solutions but with lesser resolution on the higher wave numbers.

Three different combinations of $M_{t, 0}$ and $R e_{\lambda, 0}$ have been investigated: (i) $M_{t, 0}=0.2$ and $R e_{\lambda, 0}=125.0$; (ii) $M_{t, 0}=0.6$ and $R e_{\lambda, 0}=100.0$; and (iii) $M_{t, 0}=1.5$ and $R e_{\lambda, 0}=50.0$. The lowest turbulent Mach number case is free of eddy-shocklet formation. ${ }^{38}$ The two other cases both contain eddy shocklets in the flow field. Figure 11 shows the computed isosurfaces of the Q-criterion color shaded by the local Mach number contours for the $M_{t}=1.5$ case at two different time instants. The relatively larger initial structures (see Fig. 11(a)) decay into smaller turbulent structures with a reduced speed at a later time (Fig. 11(b)). More "tubular" structures are also visible as the isotropic turbulence decays primarily due to vortex stretching. The instantaneous density gradient contours in the middle plane at the same time instance are shown in Fig. 12. Eddy shocklets are clearly visible at this turbulent Mach number. These results were obtained by using the $4^{\text {th }}$-order CESE method on a $122^{3}$ tetrahedral mesh and, and compare qualitatively well with those from Martin et al. ${ }^{40}$

The computed kinetic energy spectra, $E(k)$, at several time instances are shown in Fig. 13. The Fourier spectra were computed by interpolating the computational results onto a structured $256^{3}$ hexahedral mesh by using $3^{\text {rd }}$-order solution polynomials within each solution element. Fast Fourier Transform (FFT) was then carried out in all three directions to obtain the kinetic energy spectra shown in the figure. The shock sensor described in Ref. 7 is used to switch from a $4^{\text {th }}$-order to a $2^{\text {nd }}$-order accurate scheme at locations where shocks are detected. This approach allows shocks to be captured while maintaining higher accuracy away from the discontinuities. A CFL number around 0.8 was used for all the simulations presented here. The energy spectra results shown in Fig. 13 exhibit a clear energy containing range, an inertial subrange, and a dissipation range. The initial spectra quickly develop into a full range of turbulent spectra as shown in the figure. The decay rate in the inertial subrange exhibits a $k^{-5 / 3}$ slope, followed by the much more rapid decay into small scales at high wave numbers.

Although not shown here, spectral results obtained for all three turbulent Mach numbers exhibit similar inertial subrange and dissipation range. The $2^{\text {nd }}$-order results with the same grid show a shorter inertial subrange and more rapid decay at high wavenumbers as compared with those from the high-order results shown in Fig. 13. These initial results for a high turbulent Mach number case with the presence of eddy shocklets are promising in terms of flow fidelity, numerical stability, and computed turbulent spectra. 


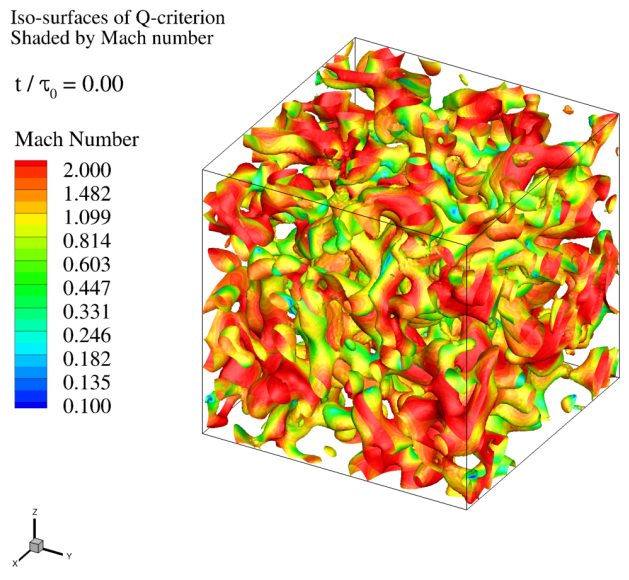

(a)

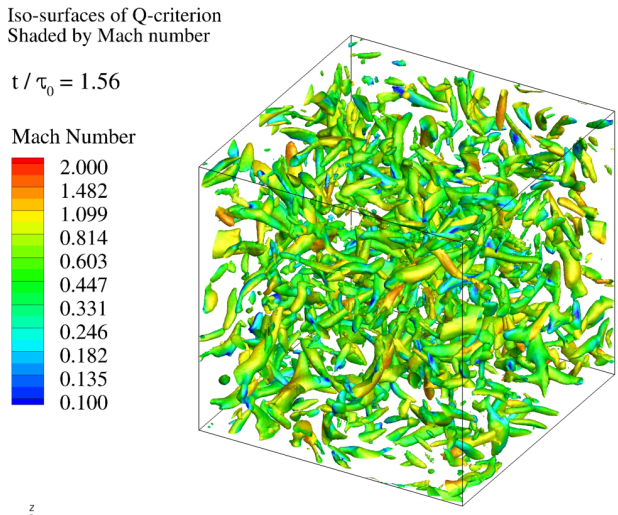

(b)

Figure 11. Computational results for isotropic turbulent flow decay in a periodic box with $M_{\mathrm{t}}=1.5$, showing isosurfaces of Q-criteria shaded by Mach numbers: (a) $t=0$ (b) $t / \tau_{0}=1.56$

$\mathrm{t} / \tau_{0}=1.56$
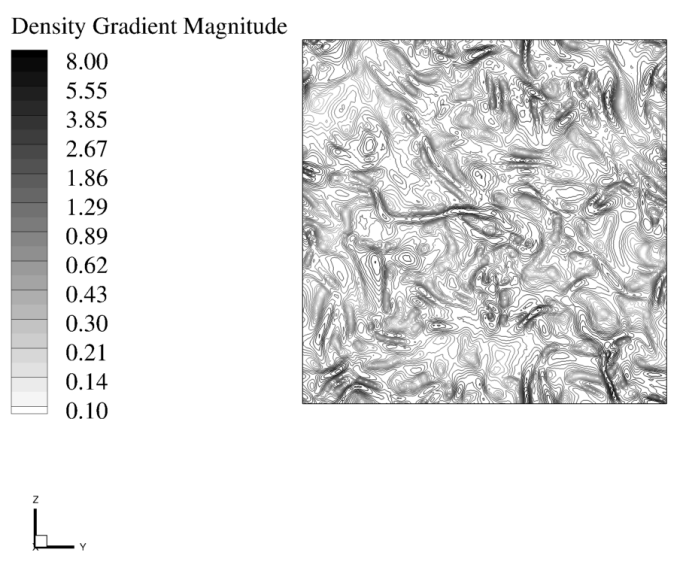

Figure 12. Computed density gradient contours for $M_{\mathrm{t}}=1.5$ at a representative plane.

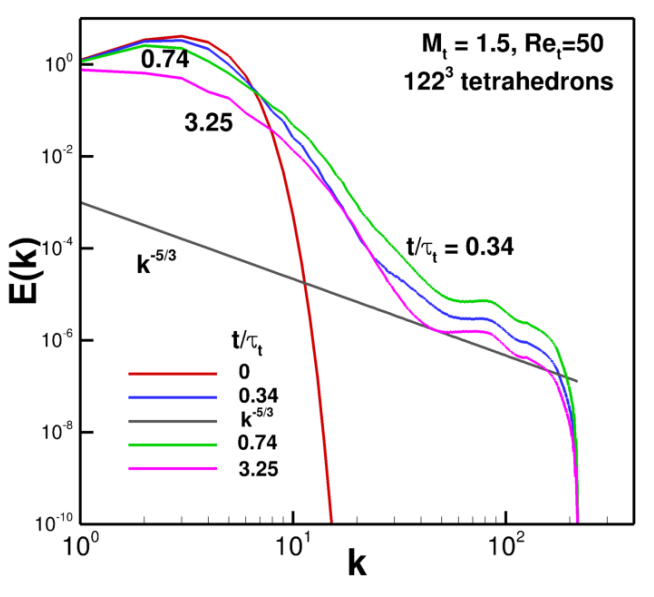

Figure 13. Computed kinetic energy spectra for $M_{\mathrm{t}}=1.5$ at several time instances.

\section{E. Canonical Isotropic Turbulence - Normal Shock Interaction}

Having obtained satisfactory results for single vortex/shock interaction and the decay of homogeneous isotropic turbulence involving high turbulent Mach numbers, the next logical step is to carry out computations of the canonical shock/turbulence interaction, which involves a broad range of scales. In this study, the focus is on the most fundamental problem in shock/turbulence interaction, namely that of isotropic turbulence passing through a nominally normal shock. To begin with, 2D computations were performed for a rectangular domain extending from 0 to $4 \pi$ in the $x$-direction (flow direction) and from 0 to $2 \pi$ in the transverse direction. The normal shock was located at $x=2 \pi$. Meshes containing isotropic triangles generated using a commercial grid generation tool are used for the solutions presented in this subsection. Several meshes were tested, with all of them having no clustering of mesh points around the shock region. Computations made with meshes containing about 85,000 (for Figs. 14(a)-14(c)), and 330,000 triangular elements (for Fig. 12(d)), respectively. At the top and bottom boundaries, periodic conditions are used. The same $M_{1}=1.2$ normal shock conditions as in the vortex/shock interaction section 
were also used here. At the inflow plane, isotropic turbulent structures as described in the previous section are superimposed but with a zero spanwise wave number $\left(k_{z}=0\right)$. The aforementioned approach of imposing inflow conditions, is adopted from earlier studies ${ }^{41-43}$ that specify the method of generating inflow turbulence for studying spatially evolving turbulence and their interaction with a shock wave. Randomization of the phase factor (used to prescribe the energy spectrum) in time, which is needed to ensure that the signal generated at the inflow is not periodic, ${ }^{43}$ has not been accounted for in this study. At the outflow boundary, subsonic conditions with a Mach number of $M_{2}=0.84$ are used without a buffer domain. In fact, all the 2D and 3D computations performed in this paper, unlike previous studies, ${ }^{41,42,44}$ did not require special treatment of the outflow boundary condition (buffer domain or sponge region to damp any acoustic reflection) or clustering of the grid points around the shock, to ensure that the shock was stationary and did not convect upstream. The space-time conservation formulation allows perturbations at the outflow boundaries to exit along the characteristic surfaces without incurring substantial spurious reflections. ${ }^{15-17}$

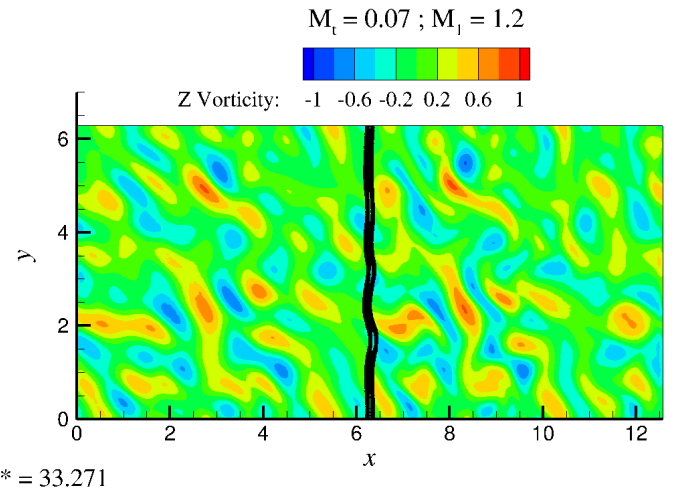

(a)

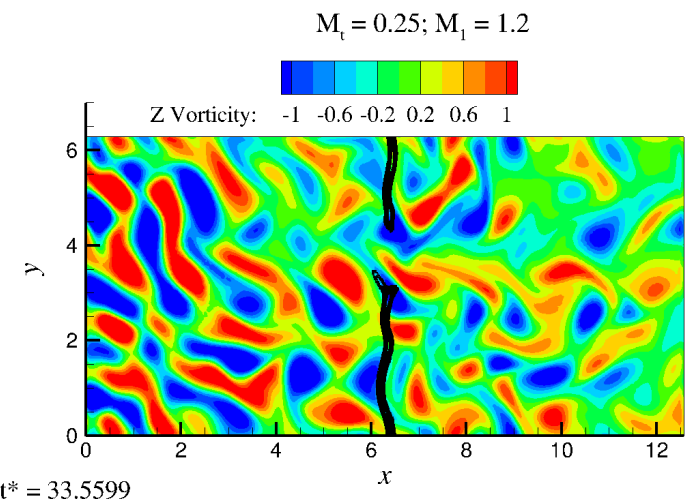

(c)

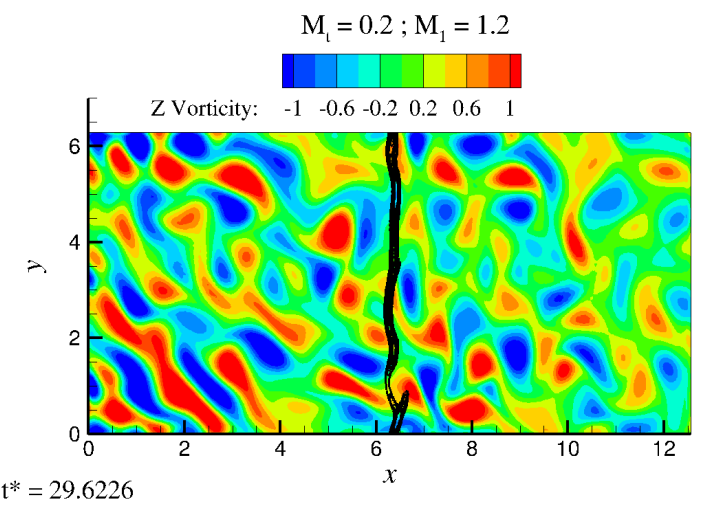

(b)

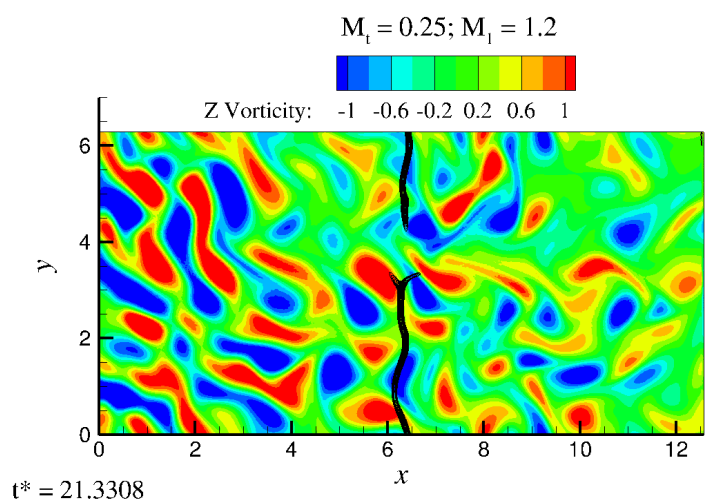

(d)

Figure 14. Contours of the $z$-vorticity for the interaction of isotropic turbulence with a normal shock $\left(M_{1}=1.2\right)$, for different turbulent Mach numbers and mesh resolution: (a) $M_{\mathrm{t}}=0.07$ (b) $M_{\mathrm{t}}=0.2$ (c) $M_{\mathrm{t}}$ $=0.25$ (d) $M_{\mathrm{t}}=0.25$ but with a finer mesh. The shock surface seen at the center of each domain is represented by contour lines of constant dilatation.

Inflow turbulence with several different turbulent Mach numbers and $R e_{\lambda, 0}=19.6$ were investigated, based on Refs. 41, 42, and 44, and the results are shown in Fig. 14. As the inflow turbulent Mach number increases, stronger vortical structures are evident both in pre- and post-shock regions. Contour lines of constant dilatation value are plotted in the same figures to indicate the instantaneous location of the shock. The normal shock starts to have strong distortion for $M_{\mathrm{t}}=0.2$ and also exhibits a small branch near the bottom. At the highest turbulent Mach number of 0.25, the shock is broken, as stronger turbulent vortical structures pass through and interact with it. These modifications of the shockwave are in line with observations from earlier DNS studies. ${ }^{42,44}$ In Fig. $14(\mathrm{~d})$, the same $M_{\mathrm{t}}=0.25$ 
simulation carried out using a mesh with approximately 330,000 triangular elements is shown. With a finer mesh, the shock gets resolved sharply, and the smaller structures in the post-shock section get resolved well with a relatively smaller core region (with red or blue colors).

Figure 15 (a) shows the time-evolution of the vortices as they pass through the shock for $M_{\mathrm{t}}=$ 0.07. Even for this low turbulence intensity level, the intensification and deformation of the vortices, as they pass through the shock, is evident from the contour plots in Fig. 15 (a) and from the post-shock amplification of the vorticity shown in Fig. 15 (b). Given the small value of the turbulent Mach number, the amplification of the $z$-vorticity square, shown in Fig. 15 (b), across the shock (about $72 \%$ ) compares with predictions from the linear theory ( $\sim 80 \%$, see Fig. 8 of Ref. 42$)$. The mean profiles of density and pressure shown in Fig. 16 clearly indicate that the jumps at the shock are smaller than those given by laminar Rankine-Hugoniot relations. The deviation from the laminar jump conditions increases with $M_{\mathrm{t}}$. Behind the shock, there is an overshoot in mean values, followed by a relaxation. Lele ${ }^{45}$ had derived the deviation of the shock-jump relations in a turbulent flow from its laminar counterpart, through utilization of rapid distortion theory for closure approximation. The mean results obtained in this study are consistent with the conclusions from Lele's study ${ }^{45}$ - increased $M_{\mathrm{t}}$ leads to smaller jumps in density and pressure for a fixed inflow Mach number.

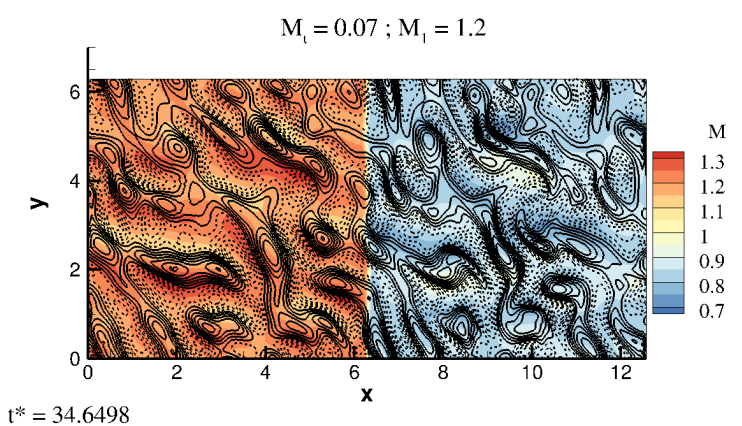

(a)

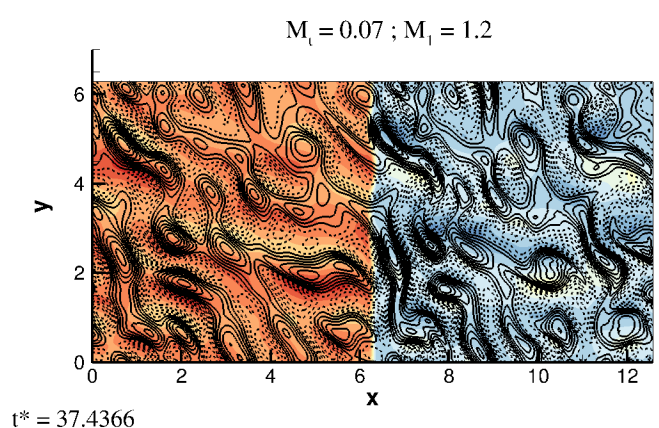

$t^{*}=37.4366$

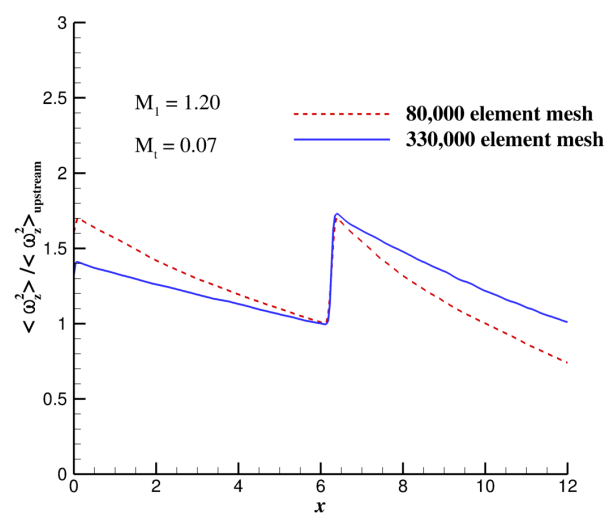

(b)

Figure 15. Shock/turbulence interaction with $M_{1}=1.2$ and $M_{\mathrm{t}}=0.07$ : (a) Snapshots of $z$-vorticity contour lines in at two different time instances. Solid (dashed) lines indicate positive (negative) vorticity. Mach contour is shown in the background; (b) Evolution of mean square vorticity. 


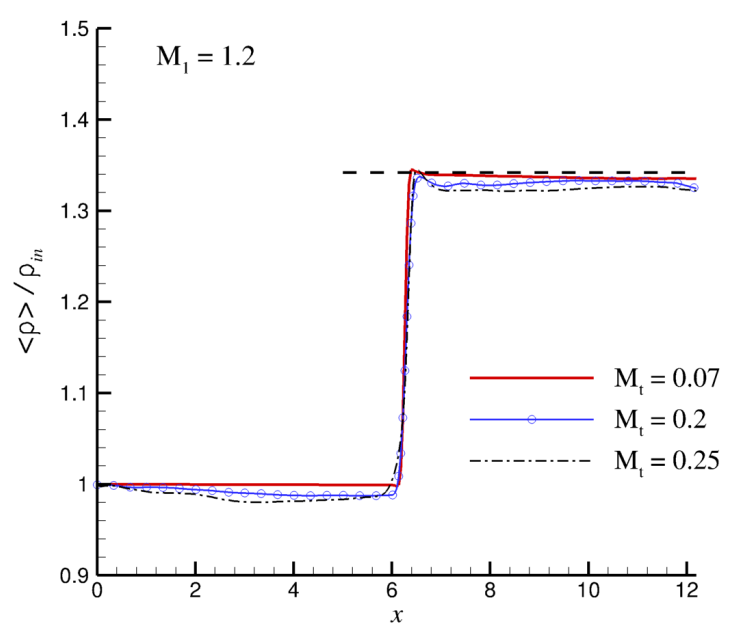

(a)

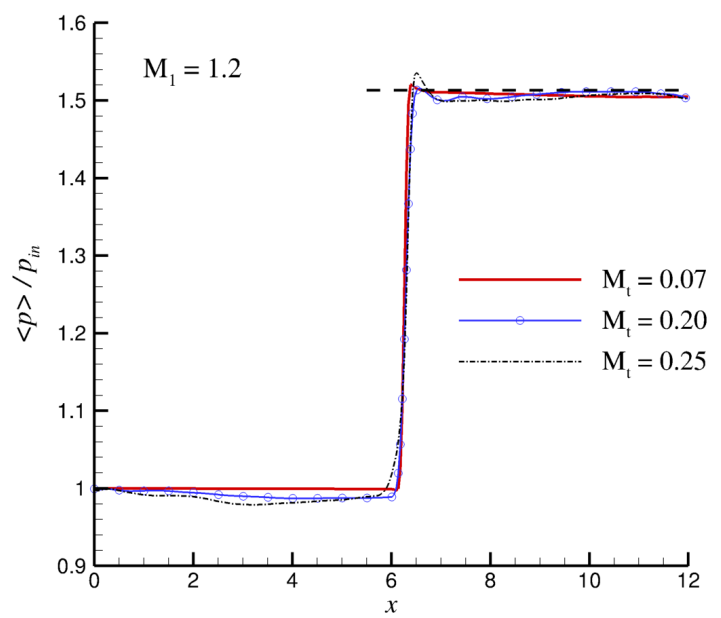

(b)

Figure 16. Mean profiles of density and pressure (normalized by their initial values at inflow) due to the interaction of turbulence and a Mach 1.2 normal shock, showing different intensities $\left(M_{t}=0.07\right.$, 0.2 , and 0.25). The dashed horizontal line denotes the laminar post-shock states: (a) mean density; (b) mean pressure.

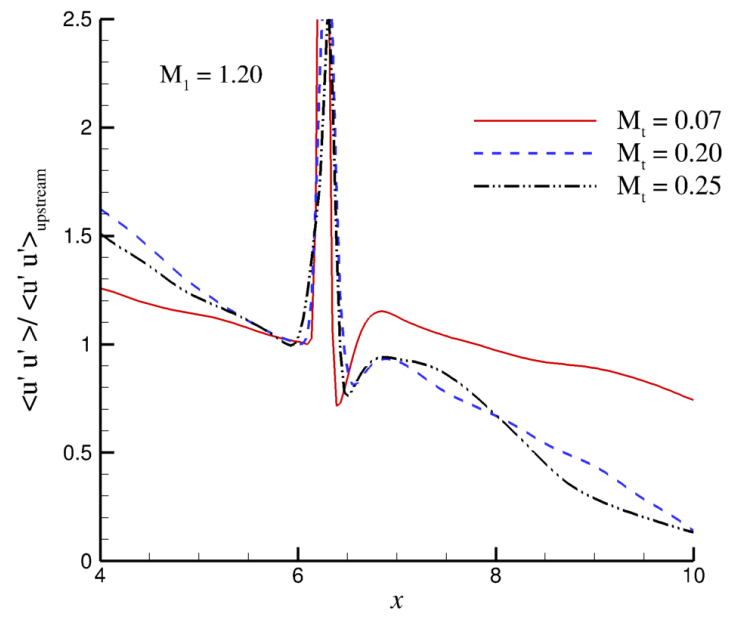

(a)

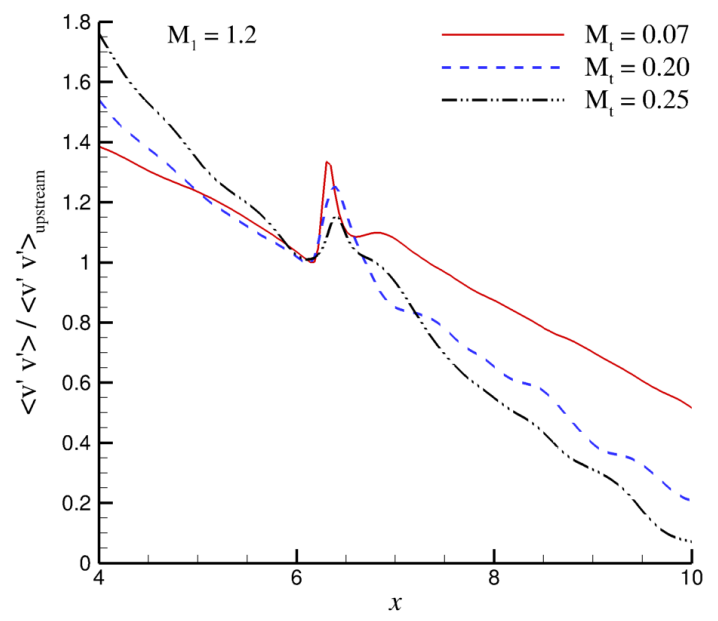

(b)

Figure 17. Influence of $M_{\mathrm{t}}$ on the evolution of the diagonal components of the Reynolds stress tensor in shock-turbulence interaction $\left(M_{1}=1.2\right)$ : (a) streamwise component; (b) transverse component.

The diagonal components of the Reynolds stresses (normalized with their values just upstream of the shock) for three different turbulent Mach numbers and an identical mean flow Mach number are compared in Fig. 17. Both streamwise and transverse Reynolds stresses increase at the shock. The large fluctuation of the streamwise component in the shock region is due to the intermittent shock front distortions. The transverse stress decays almost monotonically behind the shock, while there is a rapid increase of the streamwise component behind the shock before it begins to decay slowly. The levels of fluctuation in the streamwise component just behind the shock is in general larger than the transverse component because of the contribution from the acoustic waves that are generated when the vortical waves interact with the shock. The post-shock increase of the streamwise component of the Reynolds stress is not predicted by the linear theory ${ }^{22}$ and can only be explained by nonlinear effects. ${ }^{41,42,44}$ The behavior of the Reynolds stresses observed in this study has also been well-investigated in the literature. ${ }^{42,44}$ 


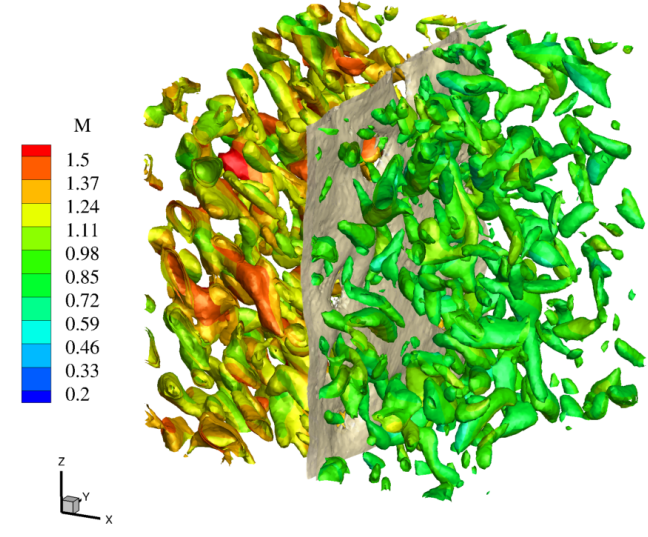

(a)

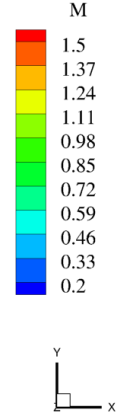

(b)

Figure 18. Solutions for interaction of isotropic turbulence $\left(M_{\mathrm{t}}=0.25\right)$ with a normal shock $\left(M_{1}=1.2\right)$, showing isosurfaces of $Q$-criterion shaded with the local Mach number. Shock surface is represented by isosurface of dilatation. (a) Side view; (b) top view ( $x-y$ plane).

The strongest interaction case with $M_{1}=1.2$ and $M_{\mathrm{t}}=0.25$ was repeated with 3D computations. A cubic domain with a side length of $2 \pi$ and 2.7 million tetrahedral elements was used in the computations. The normal shock was located at $x=\pi$. The upstream $\left(M_{1}=1.2\right)$ and downstream $\left(M_{2}=0.84\right)$ boundaries are set at $x=0$ and $x=2 \pi$, respectively. As in the $2 \mathrm{D}$ computations, the inflow plane is superimposed with isotropic turbulent structures that are convected downstream through the shock.

The instantaneous high-order solution at a non-dimensional time of 11.7 (normalized by the inverse of the freestream velocity) is shown in Fig. 18. The Q-criterion isosurfaces have been color shaded by the Mach numbers, showing pre- and post-shock regions with supersonic and subsonic colors, respectively. Isosurfaces of dilatation are used in these plots to help visualize the instantaneous shock surface in the middle of the domain. The isotropic nature of the incoming turbulence is indicated by the random orientation of the vortex cores upstream of the shock surface. The shock compresses the turbulence in the streamwise direction, distorts the vortices, and in the meantime, makes them predominantly align in the $y-z$ plane (see the top view, Fig. 18(b)). The medium large level of turbulence in the inflow causes a strong interaction with the shock surface, resulting in severe wrinkling and distortion of the shock surface, as is evident from the instantaneous shock surface shown in Fig. 19. As the shock surface in these plots is represented through isosurfaces of the dilatation, the holes that appear in the shock surface are regions of smooth isentropic compression, indicative of the broken shock that was also seen in the 2D simulations.

Based on the mean temperature profile, shown in Fig. 20(a), the 2D computations compare well with the 3D computations for this particular flow condition involving a weak normal shock wave. With the 3D case being a more realistic representation of isotropic turbulence and given the extra dimension for the vortices to evolve, there is naturally a little difference in the levels of the transverse component of the Reynolds stress, between that of the 2D and 3D computations as shown in Fig. 20(b). However, the monotonic decay of the transverse component of the Reynolds stress, downstream of the shock, is

consistent with earlier studies. ${ }^{41,42,44}$ As the computational domains of the 2D and 3D computations were of different size in the streamwise direction, the solution from the 3D computations was shifted in the $x$ direction, to facilitate the comparisons in Figs. 20(a) and (b). Although the 3D computations were computed on what is considered a coarse mesh ${ }^{44}$ by DNS standards, the computations captured the qualitative behavior of the shock-turbulence interaction. A more detailed grid-convergence study, using flow conditions studied using other numerical methods, is required to compare the performance of the CESE method against other schemes and to ensure that the error estimates in velocity and vorticity variances are within $2 \%$. Towards this end, a finer grid in the post-shock is advantageous to resolve the dissipative scales in the post-shock turbulence, as opposed to the uniform grid spacing that was used all through the computational domain in this study. With use of unstructured tetrahedral elements, this could bring about a substantial saving in the overall grid count for DNS studies, as it is free of any topological constraints. 

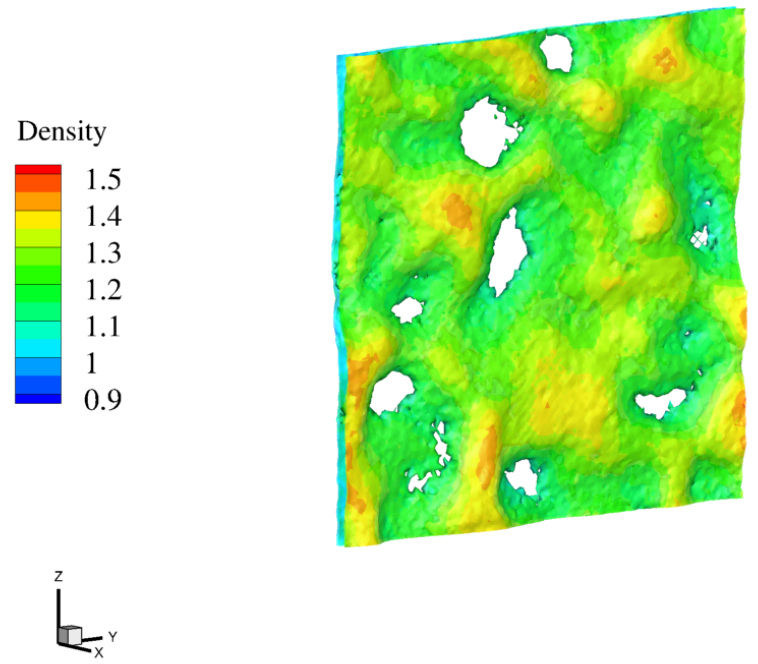

Figure 19. Wrinkled shock surface resulting from strong interaction of turbulence with a normal shock. Shock surface is represented by isosurface of dilatation shaded with density contour.

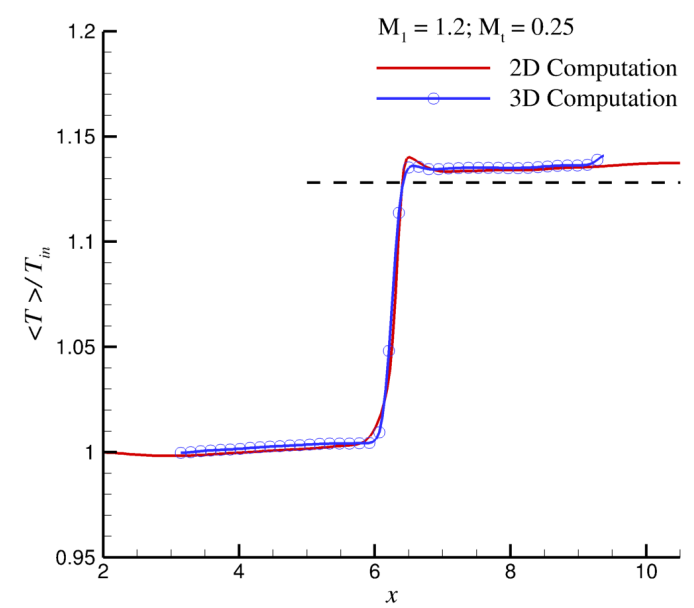

(a)

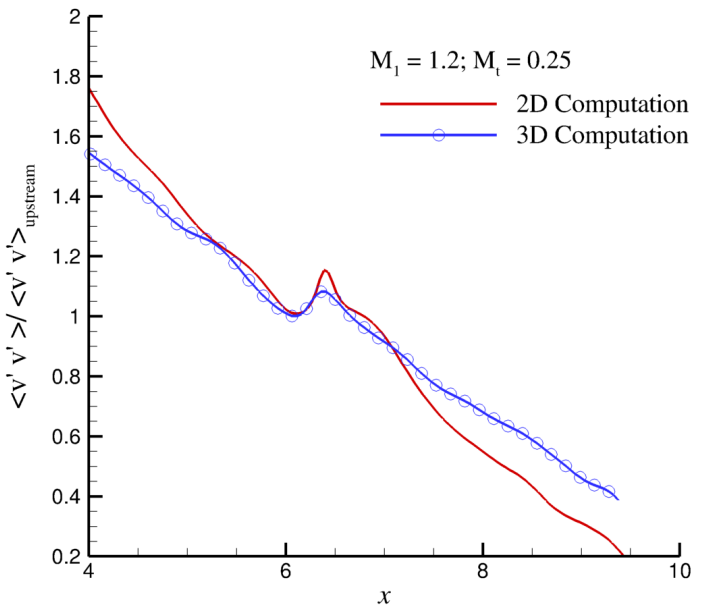

(b)

Figure 20. Comparison between two-dimensional and three-dimensional computations of shockturbulence interaction: (a) mean Temperature profile, and (b) transverse Reynolds stress component evolution.

\section{Summary}

After decades of computational fluid dynamics research and development, RANS based methods and software have matured in providing reliable state-state solutions with reasonable turnaround time. However, numerical algorithms and computational tools are still under development for efficient and accurate predictions of unsteady flows involving complex geometries and physics. The demand for higher accuracy for flows involving waves and discontinuities has been one of the main goals since the dawn of the CFD development. Traditional ways of treating shocks involve approximate Riemann solvers based on one-dimensional assumptions. Dimensional splitting in conjunction with grid adaptation is used to treat multi-dimensional shocks with reasonable accuracy. However, this approach fails to provide equally robust solutions for triangular or tetrahedral unstructured meshes. For waves and shock interaction problems, very fine mesh is usually employed around shocks to keep numerical dissipation low enough, in order not to suppress the incoming and refracted perturbations. Conventional numerical algorithms widely used to-date for DNS solve partial differential equations in all spatial directions at a fixed time level. Temporal derivatives are included in the discretized equations by an implicit or explicit finite-difference operator. 
Thus, there is an inherent assumption of smoothness in time. In contrast, the space-time CESE method formulates the space-time conservation in a unified manner. The temporal as well as spatial conservation is enforced in the discretized equations. This approach based on integral form enables the method to closely mimic the physics, and thus, allows for easy formulation of the non-reflecting boundary conditions. Potentially high-degree savings in computational time could come from the smaller domain free of buffer zones as demonstrated in the shock/vortex and shock/turbulence interaction problems discussed above.

The unstructured mesh used for isotropic turbulence simulation is free of topological and mesh clustering constraints intrinsic to the structured quadrilateral/hexahedral mesh generation. Preliminary results from the decay of isotropic turbulence simulations indicated that the expected decay rate for medium to high wave numbers can be obtained using isotropic tetrahedral meshes. More numerical investigation is needed to establish the superiority of isotropic meshes in turbulent flow simulations. For wall bounded flows, the time-accurate local time-stepping formulation implemented for the CESE method by enforcing temporal and spatial conservation can improve overall simulation efficacy when multiple physical scales exist in the computational domain; although it remains to be demonstrated. The CESE method enables a consistent numerical formulation to be employed for waves, flow discontinuities, and their interactions, free from any hybrid scheme switching that has been widely used in conventional schemes. The high-order CESE method allows local $p$-refinement capabilities with the same upper CFL bound as its low-order counterpart, a feature ideal for adaptive refinement to be implemented in the future.

\section{Acknowledgments}

The authors thank Dr. Sin-Chung Chang of the NASA Glenn Research Center for all the great technical discussions. Technical discussion with Dr. Meelan Choudhari of NASA Langley Research Center for the acoustic wave bow-shock interaction problem is greatly appreciated. The research funding for this research has been provided by the Transformational Tools and Technologies (TTT) project under the Transformative Aeronautics Concepts Program (TACP).

\section{References}

1 Bermejo-Moreno, I., Bodart, J., Larson, J., Barney, B. M., Nichols, J. W., and Jones, S., "Solving The Compressible Navier-Stokes Equations on up to 1.97 Million Cores and 4.1 Trillion Grid Points," in Proceedings of the 2013 ACM/IEEE Conference on Supercomputing (SC13), 2013.

2 Khalighi, Y., Nichols, J. W., Lele, S. K., Ham, F., and Moin, P., "Unstructured Large Eddy Simulation for Prediction of Noise Issued from Turbulent Jets in Various Configurations," AIAA Paper 2011-2886, 2011.

3 Urbin, G., and Knight, D., "Large-Eddy Simulation of a Supersonic Boundary Layer Using an Unstructured Grid," AIAA J., Vol. 39, No. 7, pp. 1288-1295, 2001.

4 Chang, S.-C., "The Method of Space-Time Conservation Element and Solution Element-A New Approach for Solving the Navier-Stokes and Euler Equations," J. Comput. Physics, Vol. 119, pp. 295-324, 1995.

5 Chang, S.-C., "A New Approach for Constructing Highly Stable High Order CESE Schemes," AIAA-2010-543, 2010.

6 Chang, C.-L., "Three-Dimensional Navier-Stokes Calculations Using the Modified Space-Time CESE Method," AIAA Paper 2007-5818, 2007.

7 Chang, C.-L., Venkatachari, B., and Cheng, G, "Time-Accurate Local Time Stepping and High-Order Space-Time CESE Methods for Multi-Dimensional Flows with Unstructured Meshes," AIAA Paper 2013-3069, 2013.

8 Chang, S.-C., Wu, Y., Yang, V., and Wang, X. Y., "Local Time-Stepping Procedures for the Space-Time Conservation Element and Solution Element Method," Inter. J. of Comput. Fluid Dyn., Vol. 19, No. 5, pp. 359-380, 2005.

9 Roe, P. L., “An Introduction to Numerical Methods Suitable for the Euler Equations," von Karman Institute for Fluid Dynamics Lecture Series: Introduction to Computational Fluid Dynamics, 1983.

${ }^{10}$ Zwart, P. J., "The Integrated Space-Time Finite Volume Method,” Ph.D. thesis, University of Waterloo, 1999.

${ }^{11}$ Yen, J. C., "Demonstration of a Multi-Dimensional Time-Accurate Local Time Stepping CESE Method," AIAA Paper 2011-2755, 2011.

12 Chang, C.-L., Choudhari, M. M., and Li, F., "Numerical Computations of Hypersonic Boundary-Layer over Surface Irregularities," AIAA Paper 2010-1572, 2010.

${ }^{13}$ Chang, C.-L., Choudhari, M., Li, F., and Venkatachari, B. S., "Effects of Cavities and Protuberances on Transition over Hypersonic Vehicles," AIAA Paper 2011-3245, 2011.

${ }^{14}$ Wang, X. Y., Chang, S.-C., "A 2D Non-Splitting Unstructured Triangular Mesh Euler Solver Based on the Space- 
Time Conservation Element and Solution Element Method," Comp. Fluid Dynamics Journal, Vol. 8, No. 2, pp. 309325, 1999.

${ }^{15}$ Loh, C. Y., Chang, S.-C., and Scott, J. R., "Computational Aeroacoustics via the Space-Time Conservation Element/ Solution Element Method," AIAA Paper 96-1687, 1996.

${ }^{16}$ Loh, C. Y., Hultgren, L. S., and Chang, S. C., "Wave Computation in Compressible Flow Using Space-Time Conservation Element and Solution Element Method," AIAA J., Vol. 39, No.5, pp. 794-801, 2001.

${ }^{17}$ Chang, S.-C., Himansu, A., Loh, C. Y., Wang, X.-Y, and Yu, S.-T., "Robust and Simple Non-Reflecting Boundary Conditions for the Euler Equations-A New Approach Based on the Space-Time CE/SE Method," NASA/TM-2003212495,2003

18 " $2^{\text {nd }}$ International Workshop on High-Order CFD Methods," DLR, AIAA and AFOSR, http://www.dlr.de/as/desktopdefault.aspx/tabid-8170/13999 read-35550/ [cited 14 May 2014].

19 Bilyeu, D. L., "A Higher-Order Conservation Element Solution Element Method for Solving Hyperbolic Differential Equations on Unstructured Meshes," PhD thesis, The Ohio State University, 2013.

${ }^{20}$ Bilyeu, D. L., Yu, S.-T. J., Chen, Y.-Y., and Cambier, J,-L., "A Two-Dimensional Fourth-Order Unstructured Meshed Euler Solver Based on the CESE Method," J. Comput. Phys., Vol. 257, pp. 981-999, 2014.

${ }^{21}$ McKenzie, J. F. and Westphal, K. O., "Interaction of Linear Waves with Oblique Shock Waves," Physics of Fluids, Vol. 11, No. 11, pp. 2350-2362, 1968.

${ }^{22}$ Anyiwo, J. C. and Bushnell, D. M., "Turbulence Amplification in Shock-Wave Boundary Layer Interaction," AIAA J., Vol. 20. No. 7, pp. 893-899, 1982.

${ }^{23}$ Chang, C.-L., Malik, M. R., and Hussaini, M. Y., "Effects of Shock on the Stability of Hypersonic Boundary Layers," AIAA Paper 90-1448, 1990.

${ }^{24}$ Lele, S. K., "Compact finite difference schemes with spectral-like resolution," J. Comput. Phys., Vol. 103, pp. 1642, 1992.

${ }^{25}$ Balsara, D., and Shu, C.W., "Monotonicity preserving weighted essentially non-oscillatory schemes with increasingly high order of accuracy," J. Comput. Phys., Vol. 160, pp. 405-452, 2000.

${ }^{26}$ Robinet, J.-Ch., Gressier, J., Casalis, G. and Moschetta, J.-M., "Shock Wave Instability and Carbuncle Phenomenon: Same Intrinsic Origin?” J. Fluid. Mech. Vol. 417, pp. 237-263, 2000

${ }^{27}$ Chauvat, Y., Moschetta, J.-M., and Gressier, J., "Shock Wave Numerical Structure and the Carbuncle Phenomenon," Int. J. Numeri. Meth. Fluids, Vol. 47, No. 8-9, pp. 903-909, 2004.

${ }^{28} \mathrm{Hu}, \mathrm{S}$., and Zhong, X., "Hypersonic Boundary-Layer Stability over Blunt Leading Edges with Bow-Shock Effects," AIAA Paper 1998-433, 1998.

${ }^{29}$ Inoue, O., and Hattori, Y., "Sound generation by shock-vortex interactions," J. Fluid Mech., Vol. 380, pp. 81-116, 1999.

${ }^{30}$ Grasso, F. and Pirozzoli, S., "Shock-Wave-Vortex Interactions: Shock and Vortex Deformations, and Sound Production," Theoret. Comput. Fluid Dynamics, Vol. 13, pp. 421-456, 2000.

${ }^{31}$ Dosanjh, D. S., and Weeks, T. M., "Interaction of a starting vortex as well as a vortex street with a traveling shock wave," AIAA J., Vol. 3, No. 2, pp. 216-223, 1965.

${ }^{32}$ Taylor, G. I., and Green, A. E., "Mechanism of the Production of Small Eddies from Large Ones," Proc. R. Soc. London A, Vol. 158, pp. 499-521, 1937.

${ }^{33}$ Brachet, M., Meiron, D. I., Orzag, S. A., Nickel, B. G., Morf, R. H., and Frisch, U., "Small-Scale Structure of the Taylor-Green Vortex," J. Fluid Mech., Vol. 130, pp. 411-452, 1983.

${ }^{34}$ Hunt, J. C. R., Wray, A. A., and Moin, P., "Eddies, stream, and convergence zones in turbulent flows," Center for Turbulence Research Report CTR-S88, 1988.

${ }^{35}$ Johnsen, E., Larsson, J., Bhagatwala, A. V., Cabot, W. H., Moin, P., Olson, B. J., Rawat, P. S., Shankar, S. K., Sjogreen, B., Yee, H. C., Zhong, X., and Lele, S. K., "Assessment of High Resolution Methods for Numerical Simulations of Compressible Turbulence,” J. Comput. Phys., Vol. 228, pp. 1213-1237, 2010.

${ }^{36}$ Shu, C.-W., Don, W.-S., Gottlieb, D., Schilling, O., and Jameson, L., "Numerical Convergence Study of Nearly Incompressible, Inviscid Taylor-Green Vortex Flow,” J. Sci. Comput., Vol. 24, No. 1, pp. 1-27, 2005.

${ }^{37}$ Swanson, R. C., Rumsey, C. L., Rubinstein, R., Balakumar, P., and Zang, T. A., "Parametric Study of Decay of Homogeneous Isotropic Turbulence Using Large Eddy Simulation," NASA/TM-2012-217593, 2012.

${ }^{38}$ Lee, S., Lele, S. K., and Moin, P., "Eddy Shocklets in Decaying Compressible Turbulence," Phys. Fluids A, Vol. 3 , pp. 657-664, 1991.

39 Ristorcelli, J. R., and Blaisdell, G. A., "Consistent Initial Conditions for the DNS of Compressible Turbulence," Phys. Fluids, Vol. 9, pp. 4-6, 1997.

${ }^{40}$ Martin, M. P., Taylor, E. M., Wu, M., and Weirs, V. G., "A Bandwidth-Optimized WENO Scheme for the Effective Direct Numerical Simulation of Compressible Turbulence,” J. Comput. Phys., Vol. 220, pp. 270-289, 2006.

${ }^{41}$ Lee, S., Lele, S. K., and Moin, P., "Direct Numerical Simulation and Analysis of Shock Turbulence Interaction," AIAA Paper 91-0523, 1991.

${ }^{42}$ Lee, S., Lele, S. K., and Moin, P., "Direct Numerical Simulation of Isotropic Turbulence Interacting with a Weak Shock Wave," J. Fluid Mech., Vol. 251, pp. 533-562, 1993.

${ }^{43}$ Lee, S., Lele, S. K., and Moin, P., "Simulation of Spatially Evolving Turbulence and the Applicability of Taylor's Hypothesis in Compressible Flow," Phys. Fluids A, Vol. 4, pp. 1521-1530, 1992. 
${ }^{44}$ Larsson, J., and Lele, S. K., "Direct Numerical Simulation of Canonical Shock/Turbulence Interaction," Phys. Fluids, Vol. 21, 126101, 2009.

${ }^{45}$ Lele, S. K., "Shock-Jump Relations in a Turbulent Flow,” Phys. Fluids A, Vol. 4, pp. 2900-2905, 1992. 